\title{
Track-Monitoring and Analyzing Machine Clearances during Wood Forwarding
}

\author{
Marie-France Jones, Mark Castonguay, Dirk Jaeger, Paul Arp* \\ Faculty of Forestry and Environmental Management, University of New Brunswick, Fredericton, Canada \\ Email: *arp2@unb.ca
}

How to cite this paper: Jones, M.-F., Castonguay, M., Jaeger, D., \& Arp, P. (2018). Track-Monitoring and Analyzing Machine Clearances during Wood Forwarding. Open Journal of Forestry, 8, 297-327.

https://doi.org/10.4236/ojf.2018.83020

Received: April 20, 2018

Accepted: July 15, 2018

Published: July 18, 2018

Copyright (ㅇ 2018 by authors and Scientific Research Publishing Inc. This work is licensed under the Creative Commons Attribution International License (CC BY 4.0).

http://creativecommons.org/licenses/by/4.0/

\begin{abstract}
This article reports on track-monitoring and analyzing machine clearances during wood forwarding across seasons and weather, using ultrasonic distance sensors in combination with time-stamped GPS xy locations, at $10 \mathrm{sec}$ intervals. The resulting data, obtained from 54 harvesting blocks, were analyzed by machine type (two wood forwarders and one grapple skidder), stand type (softwood plantation versus natural hardwood stands), month, slope, cartographic depth-to-water (DTW) classes, number of passes along track, and machine speed. For the most part, clearances were highly variable, due to passing over stumps, rocks, harvest slash, brushmats, ruts, and snow cover when present. This variability was on average greater for the lighter-weight wood forwarders than for the heavier-weight skidder, with the former mostly moving along equally spaced lines on brushmats, while the paths of the latter spread away from central wood-landing sites. In terms of trends, machines moved 1) more slowly on wet ground, 2) faster during returning than forwarding, and 3) fastest along wood-landing roads, as to be expected. Low clearances were most notable during winter on snow-covered ground, and on non-frozen shallow DTW and wet multiple-pass ground. During dry weather conditions, clearances also increased from low-pass tracks to multi-pass tracks due to repeat soil compaction of broadened tracks. These results are presented block-by-block and by machine type. Each block-based clearance frequency pattern was quantified through regression analysis and using a gamma probability distribution function.
\end{abstract}

\section{Keywords}

Wood Forwarding, GPS Tracks, Clearance Frequencies, Number of Passes, Speed, Ground Conditions, Depth-to-Water (DTW)

\section{Introduction}

Modern wood forwarding operations require heavy machines to move across 
sensitive terrain and soil conditions. In this regard, improper timing by season and weather can lead to substantive soil compression and rutting across and along flow channels, on ridge tops, through depressions with moist to wet soils. As soils become compacted and/or displaced, studies have shown that:

1) Soil porosity is reduced affecting the oxygen levels and gas distributions within the soil (Czyz, 2004; Berisso et al., 2012).

2) Increasing soil bulk density affects plant root distribution and decrease hydraulic conductivity thereby reducing plant available water while enhancing runoff and soil erosion (Horn et al., 1995; Startsev \& McNabb, 2000; Jamshidi et al., 2008);

3) Roots are damaged, thereby facilitating root rot (Grigal, 2000; Kozlowski, 2008);

4) Soil compaction has lasting effects on soil, with natural soil-structure recovery varying from a few years to decades (Prose, 1985; Brady \& Weil, 2008; Labelle \& Jaeger, 2011; Ezzati et al., 2012).

This article focuses on monitoring and examining machine-to-ground clearances in relation to number of passes, wood forwarding machine speed, and dry to wet ground conditions involving 54 harvest blocks in New Brunswick, Canada. For this purpose, two forwarders (John Deere 1110E and 1510E) and one grapple skidder (Tigercat 635D) were equipped with ultrasonic distance sensors, and GPS data loggers. The wood-forwarding operations involved retrieving logs from clear cuts, shelter-wood cuts, and commercially thinned forest plantations. The thinning operations involved laying out brushmats to reduce soil compression impacts (Labelle \& Jaeger, 2012). The dry to wet variations in ground conditions were related to changing seasons and weather, and to topography across each harvest block as revealed by way of the metric cartographic depth-to-water index (Murphy et al., 2011; White et al., 2012).

\section{Methods}

\subsection{Site Description}

This harvest-block study was spread across four ecoregions in New Brunswick, focusing on a North-western area near Saint-Quentin, and amid-western area centralized near Juniper and Dorn Ridge, as described below (Figure 1). For this study, the areas were split into three groups-Northwestern uplands (NWU), Midwestern uplands (MWU), and lowlands (LL).

The NWU study area (elevation range 230 to $450 \mathrm{~m}$ ) is located on the Chaleur uplands in northwest New Brunswick, north of Grand Falls and encompasses the both the highlands and the northern part of the Southern Uplands ecoregion. The forest cover includes tolerant hardwoods, mixed woods and forest plantations (spruce species). Dominant tree species refer to sugar maple (Acer saccharum Marsh.), balsam fir (Abies balsamea L.), yellow birch (Betula alleghaniensis Britt.), red spruce (Picea rubens Sarg.), black spruce (Picea mariana Mill.) and white spruce (Picea glauca (Moench) Voss). Mean annual air temperature from 


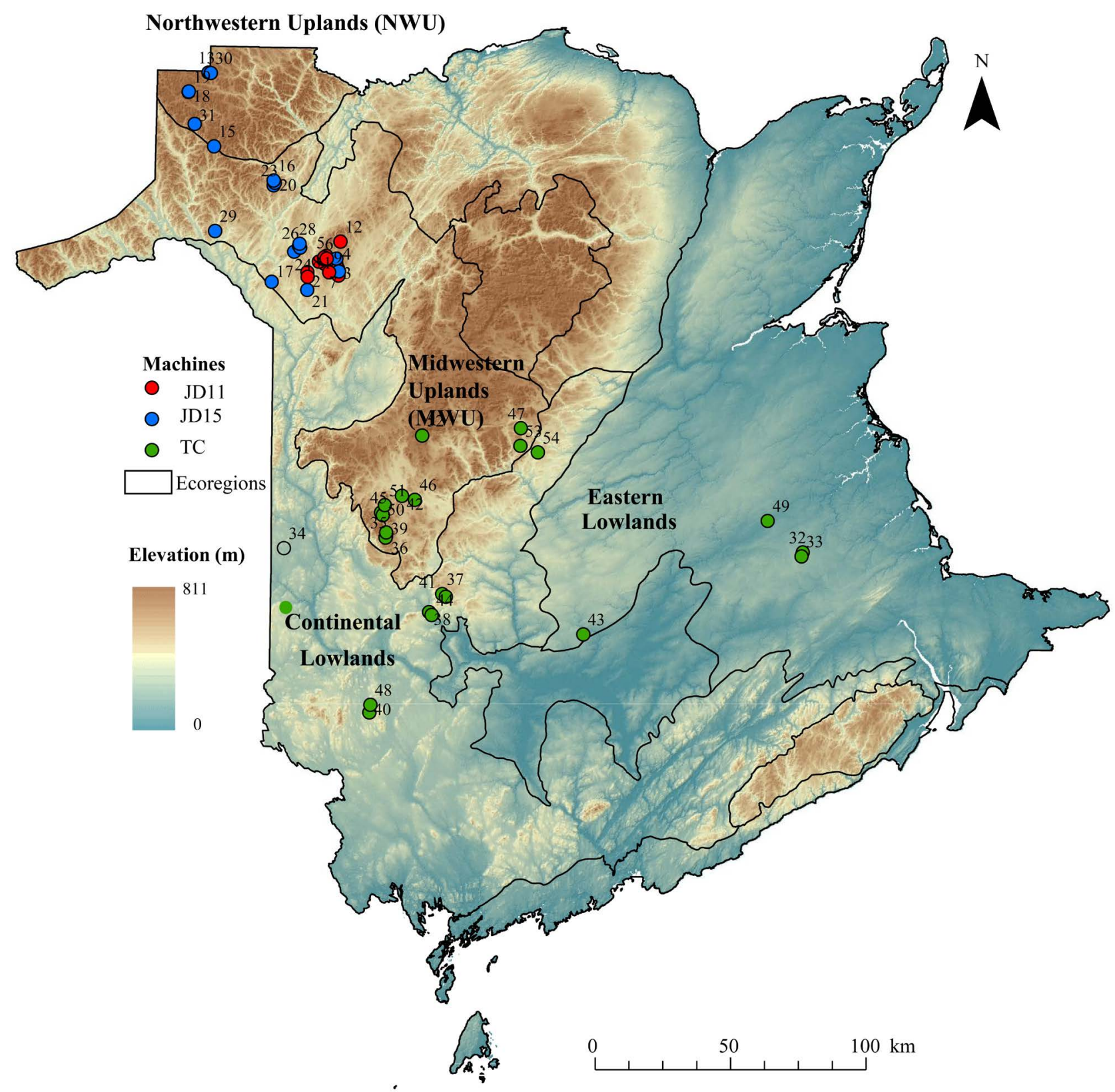

Figure 1. Map of New Brunswick showing elevations, ecoregions (GeoNB, 2018), and harvest-block locations for GPS-tracked clearances by wood-forwarding machine type (JD11, JD15, TC).

1990-2016 amounted to $3.6^{\circ} \mathrm{C}$, with mean annual January and July air temperatures at $-5.3^{\circ} \mathrm{C}$ and $12.5^{\circ} \mathrm{C}$. Mean annual precipitation was $1140 \mathrm{~mm}$, with 310 $\mathrm{mm}$ as snow (Department of Environment and Climate Change Canada, 2016). BB is underlain by Ordovician-Silurian carbonates and Ordovician-Silurian-Devonian deep-water clastics. The terrain varies from rolling to hummocky, interspersed by steeply incised valleys. Surficial deposits vary from residuals to stony ablation and loamy lodgement tills, glaciofluvial deposits (moraines, kames, eskers), and alluvium.

The MWU study range (elevations 250 to $580 \mathrm{~m}$ ) is located in mid-western 
New Brunswick, near Juniper and encompasses the lower part of the Southern Uplands ecoregion. The forest cover consists of tolerant hardwoods with sugar maple and white birch (Betula papyrifera Marsh), mixed with balsam fir and black spruce, and interspersed by black and white spruce forest plantations. Mean annual air temperature from 1990-2016 amounted to $5.2^{\circ} \mathrm{C}$, with mean annual January and July air temperatures at $-3.5^{\circ} \mathrm{C}$ and $13.9^{\circ} \mathrm{C}$. Mean annual precipitation was $1180 \mathrm{~mm}$, with $280 \mathrm{~mm}$ as snow (Department of Environment and Climate Change Canada, 2016).

The LL study range (elevations 120 to $290 \mathrm{~m}$ ) is located around $50 \mathrm{~km}$ northwest of Fredericton and covers both the Continental Lowlands and Eastern Lowlands ecoregions. This area is predominantly covered by tolerant hardwoods consisting of yellow birch, beech (Fagus grandifolia Ehrh.), and sugar maple, mixed with balsam fir, Eastern white cedar (Thuja occidentalis L.) and black spruce. The mean annual air temperature from 1990-2016 amounted to $5.5^{\circ} \mathrm{C}$, with mean annual January and July air temperatures at $-2.8^{\circ} \mathrm{C}$ and $13.8^{\circ} \mathrm{C}$. Mean annual precipitation amounted to $1100 \mathrm{~mm}$, with $250 \mathrm{~mm}$ as snow (Department of Environment and Climate Change Canada, 2016). Both MWU and LL are located on the Miramichi Caledonia highlands at the northeast stretch of the Appalachian Mountain range. Surficial deposits on rolling to moderate terrain vary from bouldery loamy lodgement till to moraines, eskers, kames and sandy glaciofluvial outwash plains. Bedrock formations on LL mainly refer to Cambrian-Ordovician-Silurian deep-water clastics, and on MWU mainly Carboniferous to Silurian felsic to mafic extrusions.

\subsection{Machinery and Sensor Installations}

In NWU, two John Deere (JD) forwarders, i.e., model JD 1110E equipped with tire chains (referred to as JD11 below), and model JD 1510E with front and back tire tracks (referred to as JD15 below) were used for tracking wood-forwarding machine clearances (Table 1, Figure 2). In MWU and LL, a Tigercat model TC 635Dgrapple skidder (referred to as TC below) was used, with chained tires in front and tracked tires in the rear. Machine clearances were tracked using custom-built data loggers (c/o FP Innovations Ltd., Montreal, Canada, Figure 3) to record time-stamped GPS locations and machine-to-ground clearances during back and forth machine travel within up to 54 harvesting blocks from February 2012 through November 2014, all at time-stamped 10-second intervals. The data loggers were installed within the operator cabs, with polycarbonate GPS antennas firmly attached to the cab roofs. Clearance tracking involved two ultrasonic sensors, each vertically placed in an open steel pipe welded or bolted to the least vulnerable position on the outside chassis of each machine, with sensor wires safely guided to the data loggers. On the two forwarders, the ultrasonic sensors were mounted on the right and left of the chassis $(145 \mathrm{~cm}$ for JD11and $140 \mathrm{~cm}$ for JD15 from the ground up to sensor). On the TC, the sensors were mounted on the front and back of the chassis $(170 \mathrm{~cm}$ and $152 \mathrm{~cm}$ from the ground to sensor, respectively). The sensors signalled machine clearances to 
Table 1. Machine specifications.

\begin{tabular}{|c|c|c|c|c|c|c|}
\hline \multirow{2}{*}{ Machine Specs } & \multicolumn{2}{|c|}{ JD11 } & \multicolumn{2}{|c|}{ JD15 } & \multicolumn{2}{|c|}{$\mathrm{TC}$} \\
\hline & Front & Rear & Front & Rear & Front & Rear \\
\hline Vehicle Weight (ton) & \multicolumn{2}{|c|}{16.5} & \multicolumn{2}{|c|}{17.3} & \multicolumn{2}{|c|}{21.4} \\
\hline Full Load (ton) & \multicolumn{2}{|c|}{11} & \multicolumn{2}{|c|}{12} & \multicolumn{2}{|c|}{15} \\
\hline Chassis Clearance, $\mathrm{M}_{0}(\mathrm{~cm})$ & \multicolumn{2}{|c|}{60} & \multicolumn{2}{|c|}{60.5} & \multicolumn{2}{|c|}{63.5} \\
\hline $\begin{array}{l}\text { Sensor to solid-ground } \\
\text { clearance, } \mathrm{S}_{0}(\mathrm{~cm})\end{array}$ & \multicolumn{2}{|c|}{145} & \multicolumn{2}{|c|}{139.7} & 170.2 & 152.4 \\
\hline Wheel Rim (cm) & \multicolumn{2}{|c|}{67.3} & \multicolumn{2}{|c|}{67.3} & \multicolumn{2}{|c|}{81.3} \\
\hline Number of Wheels & 4 & 4 & 4 & 4 & 2 & 4 \\
\hline Tire type & $710 / 45$ & -26.5 & $710 / 4$ & -26.5 & $35.5 \mathrm{~L} \times 32$ & $30.5 \mathrm{~L} \times 32$ \\
\hline Accessories & Chains & Tracks & Tracks & Tracks & Chains & Tracks \\
\hline Diameter $(\mathrm{cm})$ & \multicolumn{2}{|c|}{134.1} & \multicolumn{2}{|c|}{134.1} & 201.2 & 184.4 \\
\hline Section Height $(\mathrm{cm})$ & \multicolumn{2}{|c|}{33.4} & \multicolumn{2}{|c|}{33.4} & 59.9 & 51.6 \\
\hline Width $(\mathrm{cm})$ & \multicolumn{2}{|c|}{71.1} & \multicolumn{2}{|c|}{71.1} & 90.2 & 77.5 \\
\hline Pressure (max, psi) & \multicolumn{2}{|c|}{32} & \multicolumn{2}{|c|}{32} & \multicolumn{2}{|c|}{32} \\
\hline Foot print area $\left(\mathrm{m}^{2}\right)^{\mathrm{a}}$ & 2.1 & 4.2 & & & 1.7 & 6.1 \\
\hline Foot print pressure $(\mathrm{atm})^{\mathrm{a}}$ & 0.63 & 0.32 & & & 1.2 & 0.24 \\
\hline \multicolumn{7}{|c|}{$\begin{array}{l}\text { Soil compression }(\mathrm{cm})^{\text {a }} \text { : well-drained soil; } 50 \% \text { sand; dry to moist at field capacity } \\
\text { ( } \approx \text { plastic limit); not frozen; bulk density }=1.25 \mathrm{~g} \cdot \mathrm{cm}^{3} ;\end{array}$} \\
\hline 1 pass & $4-7 \mathrm{~cm}$ & $2-5 \mathrm{~cm}$ & $3-c$ & $\mathrm{~cm}^{\mathrm{b}}$ & $5-9 \mathrm{~cm}$ & $2-4 \mathrm{~cm}$ \\
\hline 10 passes & $8-19 \mathrm{~cm}$ & $5-11 \mathrm{~cm}$ & $7-1$ & $\mathrm{~cm}^{\mathrm{b}}$ & $10-22 \mathrm{~cm}$ & $4-7 \mathrm{~cm}$ \\
\hline 100 passes & $20-50 \mathrm{~cm}$ & $8-20 \mathrm{~cm}$ & $16-$ & $\mathrm{cm}^{\mathrm{b}}$ & $20-62 \mathrm{~cm}$ & $7-16 \mathrm{~cm}$ \\
\hline \multicolumn{7}{|l|}{ Sensor Installation } \\
\hline Number of Data Points & \multicolumn{2}{|c|}{ 26-Oct-12 } & \multicolumn{2}{|c|}{$25-$ Oct-12 } & \multicolumn{2}{|c|}{ 14-May-12 } \\
\hline 2012 & 258 , & 578 & 232 & & 382 & 652 \\
\hline 2013 & 589 , & 146 & 188 & & 789 & 204 \\
\hline 2014 & - & & & & 778 & 562 \\
\hline
\end{tabular}

${ }^{a}$ Derived from the above machine specifications, and using the methods described in Balland et al. (2008), Vega-Nieva et al. (2009), and Jones and Arp (2017). ${ }^{\mathrm{b}}$ Fully loaded.

ground, stumps, protruding rocks and brush piles directly below the sensors, adjacent to the tracks (Figure 4).

Operations involved JD11 and JD15 wood forwarding from commercially thinned forest plantations in northwest NB, mostly centred on NWU, and TC wood forwarding from shelter-wood and clear cutting operations in central west NB, mostly centered on MWU and LL. The commercial thinning operations involved laying out brushmats (Labelle \& Jaeger, 2012). The shelter-wood and clear cutting operations proceeded on bare ground covered by organic forest floor accumulations about 5 to $10 \mathrm{~cm}$ thick. JD15 operations were clearance 

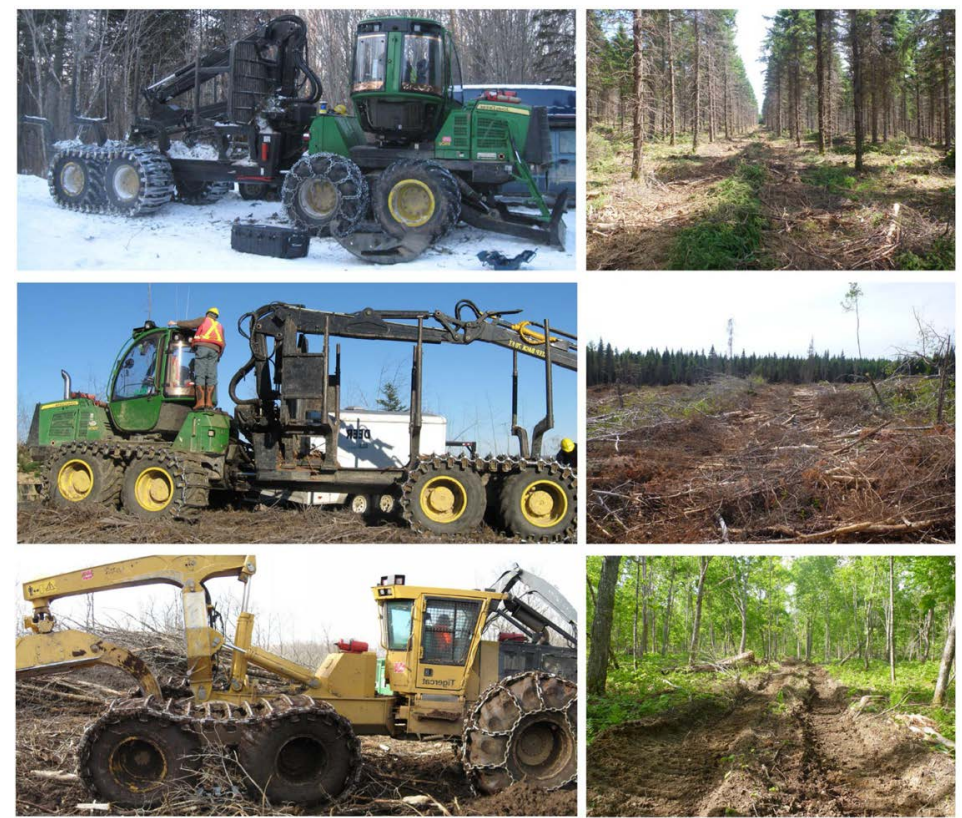

Figure 2. Left; wood-forwarding equipment: JD 1110E (top), JD 1510E (middle), and TC 635D (bottom). Right; wood harvesting-forwarding operations: commercial thinning (white spruce plantation, top), clearcutting (middle), hardwood shelter-wood cutting (bottom).
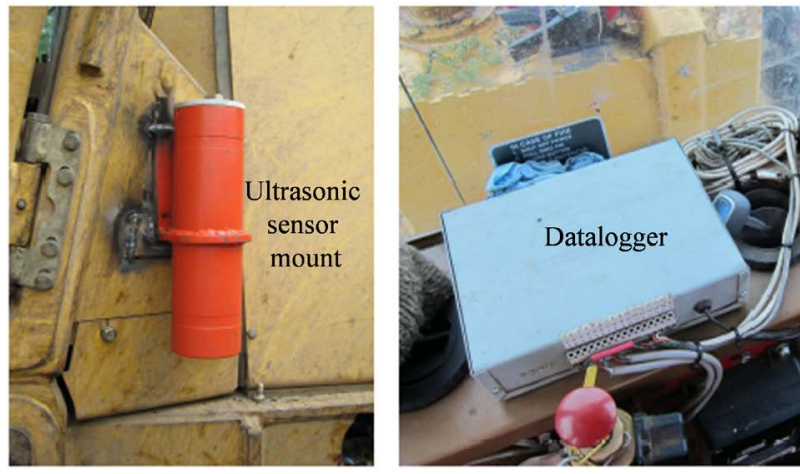

Ultrasonic distance sensor:

Measures clearance distance

from soil to chassis.

GPS sensor:

Determines XY location

Datalogger:

Records and time stamps clearance and xy location every 10 seconds.

Figure 3. Left: Ultrasonic protective mount for the ultrasonic distance sensors. Right: datalogger box connected to GPS and clearance sensors.

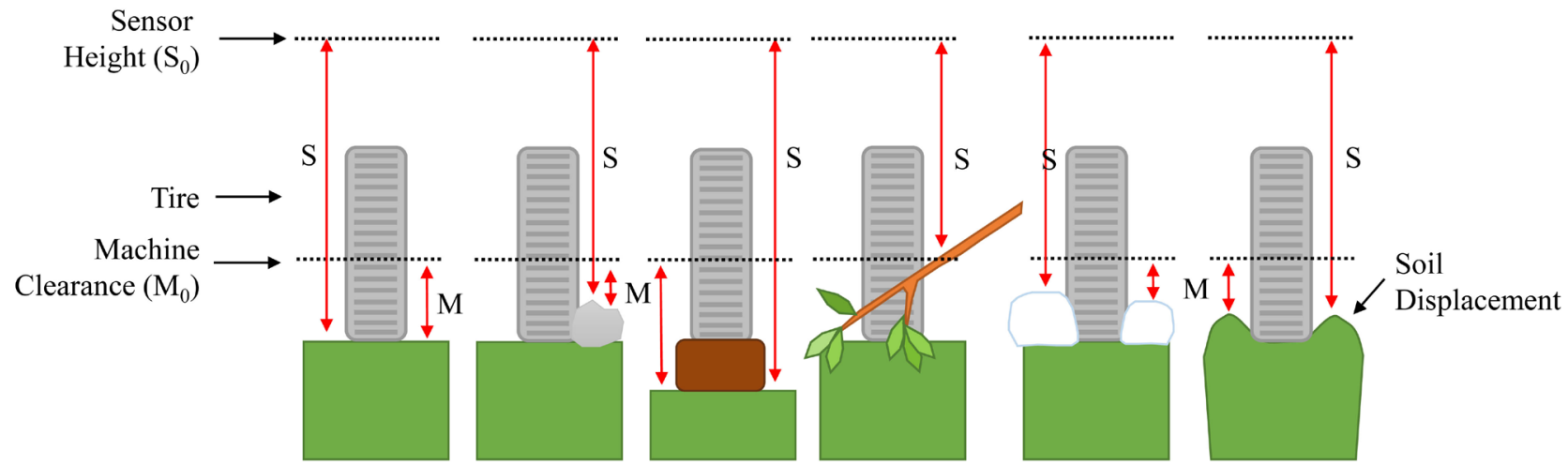

Figure 4. Various sensor distance measurements and error potential from the ultrasonic distance sensor. Left to right: rutting due to soil compaction, protruding rocks, stumps, branches and slash, and soil displacement. 
monitored year-round, including operations on snow accumulations. The JD11 and TC operations were monitored spring to fall.

\subsection{Data Production}

The logged data generated over 4.1 million data points across 54 harvesting blocks (Table 2). Data due to sensor malfunction, fixed distance-to-ground obstructions, machine idling, or traveling on paved and unpaved roads were removed. The remaining data were entered to determine point-for-point machine elevation $(\mathrm{m})$, direction, slope $(\mathrm{m} / \mathrm{m})$, and speed $(\mathrm{m} / \mathrm{sec})$ along each track. Data were catalogued for each forwarding (loaded) and returning (unloaded) pass towards the loading zones. The number of passes per same forwarding and returning track was also determined. All data were processed through ArcGIS, which also included determining track densities and number of passes per same track using point buffering and overlapping tools (Buja, 2012). The sensor-to-ground distance data $(S)$ were normalized relative to the sensor-to-ground distance on solid ground $\left(\mathrm{S}_{0}\right)$, referred to below as normalized sensor clearances $\left(S / S_{0}\right)$, or normalized clearances for short. These numbers can be converted into actual machine clearances $(M)$ by setting $M=S-\left(S_{0}-M_{0}\right)$, where $M_{0}$ is the machine-tosolid-ground (chassis) clearance (Table 1; Figure 4). Hence, at zero machine clearance $(M=0), S / S_{0}=1-M_{0} / S_{0}$. For tracks with $S<S_{0}-M_{0}$, machines bottoms would have sunk below the signal reflecting surface, as would be the case when the machines grind into wet soil or move across deep snow accumulations.

\subsection{DTW Delineation}

Each data point was placed into its geospatial elevation context using digital elevation models (DEMs) and associated depth-to-water maps (DTW, Figure 5; Murphy et al., 2009). The DTW map delineates the extent of the least elevation rise next to the nearest water bodies such as streams, rivers, and lakes (Murphy et al., 2009; White et al., 2012). The DTW map, when derived for the end-of-summer water level for water bodies emulates soil drainage across the mapped areas from very poor (DTW $<10 \mathrm{~cm}$ ), to poor $(10<$ DTW $<25 \mathrm{~cm}$ ), imperfect $(25<$ DTW $<50 \mathrm{~cm})$, moderate $(50<$ DTW $<100 \mathrm{~cm})$, well $(1<$ DTW $<20 \mathrm{~m}$ ) and excessive (DTW $>20 \mathrm{~m}$ ) (Murphy et al., 2009). The end-of-summer DTW condition was emulated using 4 ha of upslope flow accumulation area for

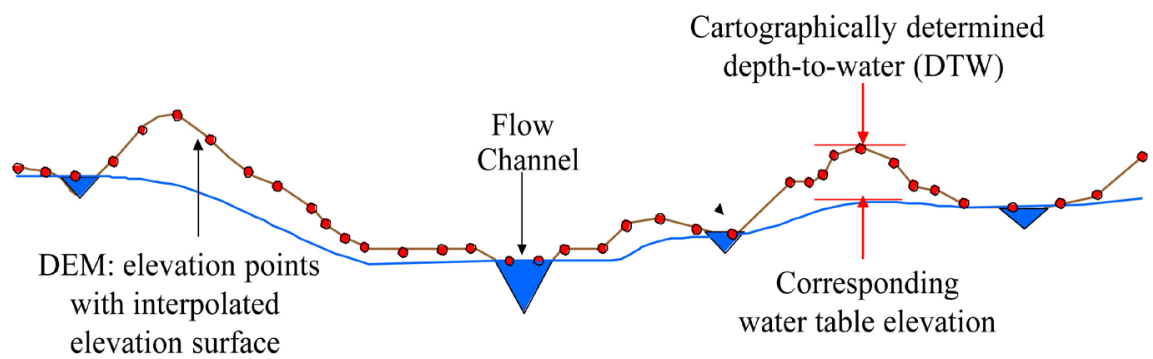

Figure 5. Cartographic depth-to-water index (DTW) diagram (Ogilvie, 2017). 
Table 2. Block description by machine, forest, operation, operation date, and ocular DTW \% area coverage.

\begin{tabular}{|c|c|c|c|c|c|}
\hline \multirow{2}{*}{ Machine } & Block & Forest & \multirow{2}{*}{ Operation } & \multirow{2}{*}{ Operation Date } & \multirow{2}{*}{ DTW \% } \\
\hline & ID & Type & & & \\
\hline \multirow[t]{12}{*}{ JD11 } & 1 & SW-BS & CT & 14-Jan & 0.95 \\
\hline & 2 & SW-WS & $\mathrm{CT}$ & Apr-May/14 & 0.5 \\
\hline & 3 & SW-WS & $\mathrm{CT}$ & 14-Aug & 0.4 \\
\hline & 4 & SW-WS & $\mathrm{CT}$ & 14-Aug & 0.3 \\
\hline & 5 & SW-WS & $\mathrm{CT}$ & $12-\mathrm{Nov}$ & 0.25 \\
\hline & 6 & SW-WS & CT & Mar-May/14 & 0.2 \\
\hline & 7 & SW-WS & $\mathrm{CT}$ & Aug-Sep/12 & 0.15 \\
\hline & 8 & SW-WS & $\mathrm{CT}$ & May-Jun/14 & 0.1 \\
\hline & 9 & SW-BS & $\mathrm{CT}$ & 14-Jun & 0.05 \\
\hline & 10 & SW-WS & CT & Jul-Aug/14 & 0.05 \\
\hline & 11 & SW-WS & $\mathrm{CT}$ & 14-Jul & 0.05 \\
\hline & 12 & SW-WS & $\mathrm{CT}$ & 12-Dec & 0.01 \\
\hline \multirow[t]{18}{*}{ JD15 } & 13 & SW-BF & $\mathrm{CC}$ & Nov-Dec/12 & 0.95 \\
\hline & 14 & SW-BS & $\mathrm{CT}$ & Jan-Feb/13 & 0.7 \\
\hline & 15 & MW-BF & SHW & 12-Dec & 0.6 \\
\hline & 16 & SW-SPBF & $\mathrm{CC}$ & 14-May & 0.5 \\
\hline & 17 & Unk & CT & Jan-Feb/13 & 0.4 \\
\hline & 18 & SW-BF & $\mathrm{CC}$ & Nov-Dec/12 & 0.4 \\
\hline & 19 & SW-BF & $\mathrm{CC}$ & 12-Dec & 0.4 \\
\hline & 20 & HW-WB & $\mathrm{CC}$ & 14-Jun & 0.25 \\
\hline & 21 & MX & $\mathrm{CC}$ & 13-Jan & 0.2 \\
\hline & 22 & SW-RP & $\mathrm{CT}$ & Oct-Nov/12 & 0.1 \\
\hline & 23 & HW-WB & SHW & 14-Jun & 0.1 \\
\hline & 24 & SW-BF & $\mathrm{CT}$ & May-June/14 & 0.1 \\
\hline & 25 & SW-BF & $\mathrm{CT}$ & May-June/14 & 0.1 \\
\hline & 26 & SW-BF & $\mathrm{CT}$ & 14-Jun & 0.1 \\
\hline & 27 & Unk & $\mathrm{CT}$ & $12-\mathrm{Nov}$ & 0.05 \\
\hline & 28 & HW-SM & SHW & 13-Jan & 0.05 \\
\hline & 29 & HW-BI & SHW & Dec/12-Jan/13 & 0.05 \\
\hline & 30 & SW-BF & $\mathrm{CC}$ & Nov-Dec/13 & 0.05 \\
\hline \multirow[t]{5}{*}{$\mathrm{TC}$} & 32 & MX-PO & $\mathrm{CC}$ & 14-Sep & 0.75 \\
\hline & 33 & MX-PO & $\mathrm{CC}$ & 14-Sep & 0.75 \\
\hline & 34 & Unk & $\mathrm{CC}$ & Jul-Aug/13 & 0.7 \\
\hline & 35 & HW-MA & SHW & 12-Jul & 0.35 \\
\hline & 36 & HW-MA & SHW & 12-Jul & 0.35 \\
\hline
\end{tabular}




\section{Continued}

\begin{tabular}{ccccc}
\hline 37 & HW-SM & SHW & 14-Oct & 0.25 \\
38 & SW-NS & CC & 13 -Jul & 0.2 \\
39 & HW-MA & SHW & 12 -Jul & 0.2 \\
40 & Unk & CC & $14-$-Jul & 0.15 \\
41 & HW-SM & SHW & Sep-Oct/14 & 0.15 \\
42 & Unk & CC & 13-Aug & 0.15 \\
43 & HW-BI & CC & 14-Sep & 0.1 \\
44 & SW-NS & CC & 13-Jun & 0.1 \\
45 & HW-MA & SHW & Aug-Sept/12 & 0.1 \\
46 & HW-MA & SHW & Oct-Nov/13 & 0.1 \\
47 & SW-RS & CT & $13-$ Aug & 0.1 \\
48 & Unk & CC & Jun-Jul/14 & 0.05 \\
49 & MX-PO & CC & Aug-Sep/14 & 0.05 \\
50 & HW-MA & SHW & Sep-Oct/12 & 0.05 \\
51 & SW-NS & CC & 12 -Aug & 0.05 \\
52 & Unk & SHW & 14-Jun & 0.05 \\
53 & HW-BE & SHW & Jun-Aug/13 & 0.05 \\
54 & HW-BE & SHW & 13-Jun & 0.05 \\
\hline
\end{tabular}

SW: Softwood, HW: Hardwood, MX: Mixedwood, Unk: Unknown, BF: Balsam Fir, WS: White Spruce, BS: Black Spruce, NS: Norway Spruce, RS: Red Spruce, WB: White Birch, BI: Birch, BE: Beech, SM: Sugar Maple, MA: Maple, PO: Poplar, CC: Clear cut, CT: Commercial Thinning; SHW: Shelterwood cut.

permanent stream flow initiation. For visualizing the varying DTW extent away from local flow channels by weather and season, ephemeral stream flow was initiated using 1 and 0.25 ha of upslope flow accumulation areas.

\subsection{Hydrological Modelling}

For contextual evaluation purposes, daily temporal variations in upland soil moisture, snowpack depth and frost depth were simulated for the NWU and for the combined MWU and LL areas using weather records for daily precipitation (rain, snow) and air temperature (daily means) from September 2011 to end of 2014 from Edmundston and Fredericton weather stations (Department of Environment and Climate Change Canada, 2016) (Appendix A3-1). Soil properties were set as follows: NWU = soil depth $1.5 \mathrm{~m}$, loam to sandy loam, organic matter content $5 \%-1 \%$, coarse fragment content $20 \%$ - 30\%; forest floor depth 10 $\mathrm{cm} ; \mathrm{MWU}=$ soil depth $1.2 \mathrm{~m}$, sandy loam, organic matter content $1 \%-10 \%$, coarse fragment $25 \%$, forest floor depth $5 \mathrm{~cm}$. LL = soil depth $1.0 \mathrm{~m}$, loamy sand, organic matter content $1 \%-5 \%$, coarse fragment $30 \%$, forest floor depth $10 \mathrm{~cm}$.

\subsection{Data Processing}

For geospatial visualization purposes, the individual data points per harvest 
block were mapped on top of the hill-shaded bare-earth DEM, with DTW drainage classes overlaid. The normalized clearance data were compiled into a single spreadsheet to enable the machine clearance analysis in relation to: 1) month of harvesting, 2) weather-induced soil wetness, 3) machine speed, 4) machine load, 5) back and forth track direction, 6) number of passes, 7) machine type, 8) harvesting type (clear cutting, commercial thinning, shelter-wood cutting), 9) slope, and 10) DTW. This was done for each harvest block to allow for detailed per block analyses in terms of 1) histogram and frequency distribution assessment, and 2) to determine how the clearance data were affected by pass number, machine speed, and by DTW classes.

\subsection{Histogram and Frequency Distribution Assessment}

The histograms for the normalized clearance data were clustered about 1 , with values $>1$ trailing off sharply, while values $<1$ trailed off slowly towards 0 , following a probability distribution function (pdf) given by (Devore, 1999):

$$
\operatorname{pdf}=\frac{1}{\Gamma(k) \theta^{k}} x^{k-1} \mathrm{e}^{-x / \theta}
$$

where $x=\left(c_{\max }-c\right) / f$, for which $c_{\max }$ is the maximum normalized clearance value (range of $0.2-0.6), \Gamma(k)$ is the gamma function, $k$ is its shape parameter, and $\theta$ and $f$ are scale parameters for $x$ and $c$, respectively. For the pdf non-linear least-squares fitting process, $c_{\max }, k, \theta$ and $f$ were used as adjustable parameters, with $\mathrm{f}$ kept in common across all blocks. Using Equation 1 implies the following properties for $\left(c_{\max }-c\right) / f:$ mean $=k \theta$, variance $=k \theta^{2}$, skewness $=2 / k^{0.5}$, mode for $k \geq 1=(k-1) \theta$ (Devore, 1999). Hence, increasing $k$ and $\theta$ implies widening the distribution function.

\subsection{Multiple Regression Analysis}

The normalized clearance data with $\leq 1$ were summarized by way of a pivot table using the following class specifications:

1) speed $(\mathrm{m} / \mathrm{sec})$

2) number of passes $(10,20,30$, etc.);

3) $\operatorname{DTW}(1=0-0.5 \mathrm{~m}, 2=0.5-1 \mathrm{~m}, 3=1-2 \mathrm{~m}, 4=2-4 \mathrm{~m}, 5=4-8 \mathrm{~m}, 6=8$ $-16 \mathrm{~m}, 7=16-32 \mathrm{~m}, 8 \geq 32 \mathrm{~m}$ );

4) forwarding (1) versus returning (0).

The data so assembled were examined using multivariate regression analysis, using the $\leq 1$ normalized clearance data as dependent variables, and number of passes, speed, and DTW classes as independent variables, by harvest block. Other topographic derivatives were analyzed (elevation, slope, aspect), but were found to be insignificant in comparison to the machine parameters.

\section{Results and Discussion}

\subsection{Wood-Forwarding Track Patterns}

Figures 6-8 show the wood forwarding and end-of-summer drainage (DTW) 


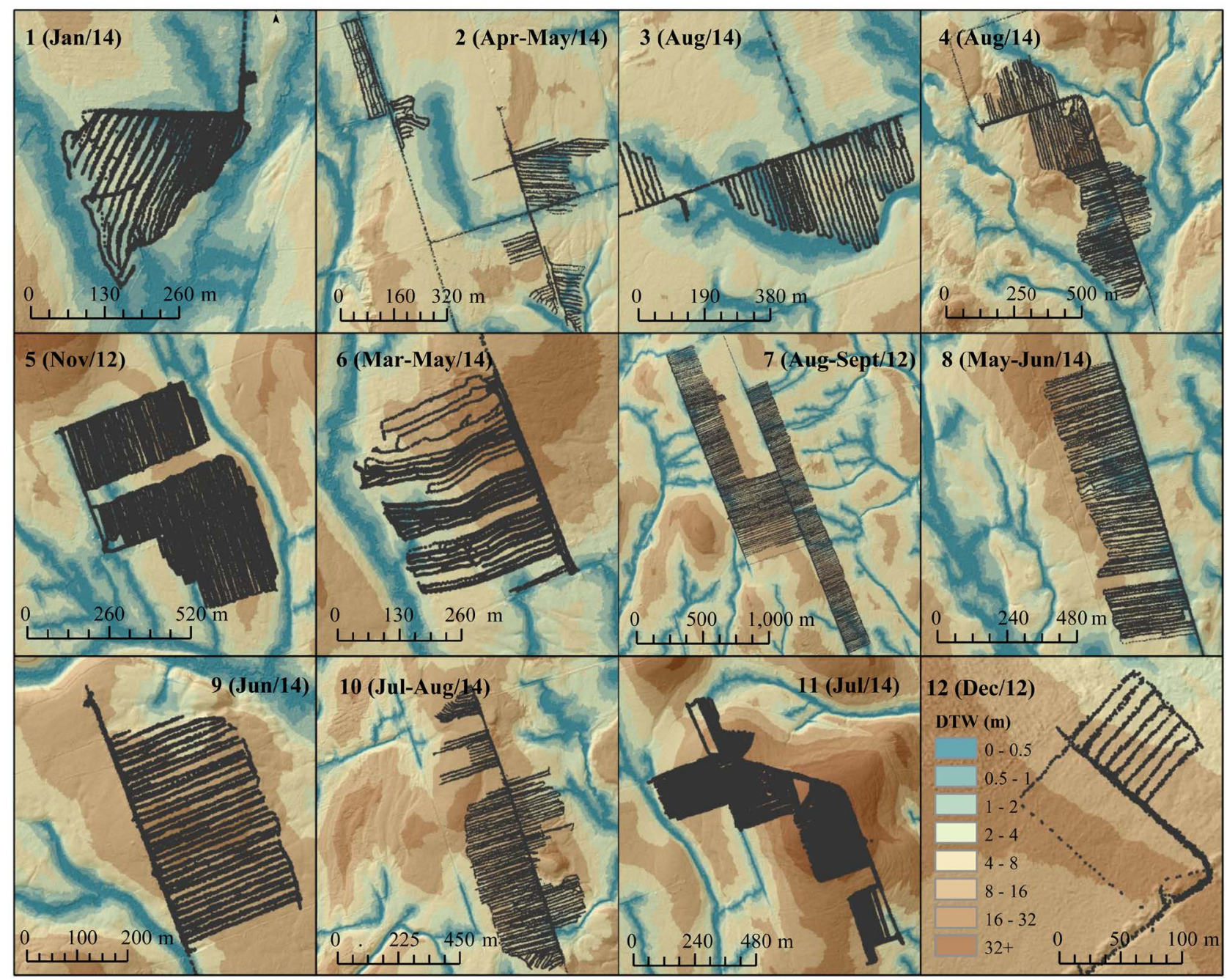

Figure 6. Wood-forwarding track patterns within JD11 harvest blocks, showing clearance sensor points overlaid on the DEM-derived DTW patterns, based on 4 ha upslope flow-initiation areas.

patterns per harvest block, by machine type. As mapped, drainage varied in area by block from poor to imperfect (DTW $<0.5 \mathrm{~m}$ ), and moderate $(0.5<\mathrm{DTW}<1$ $\mathrm{m}$ ) to well drained (DTW $>1 \mathrm{~m}$ ). Within the DTW $<0.5 \mathrm{~m}$ zone, soils were generally wet to moist. Within the $0.5<\mathrm{DTW}<2 \mathrm{~m}$ zone, soil wetness tended to be transitional from wet to dry depending on extent and weather-dependent upslope water seepage. Some of the main multiple-pass TC wood-forwarding tracks straddled across these transitions, and were rutted extensively.

The average number of passes, as well as speed, and normalized clearance are listed in Table 3. Typically, machine clearance increased towards 1 with increasing pass numbers along the same track regardless of loading. However the initial unloaded passes decreased the clearances the most (Figure 9). Conversely, machine speeds were significantly faster when empty than when loaded ( $p=$ $<0.001)$. By machine type, wood forwarding speed was lower for JD11 and JD15 than for TC, while clearances increased with increasing machine speed, and especially so for JD11 (Figure 9). 


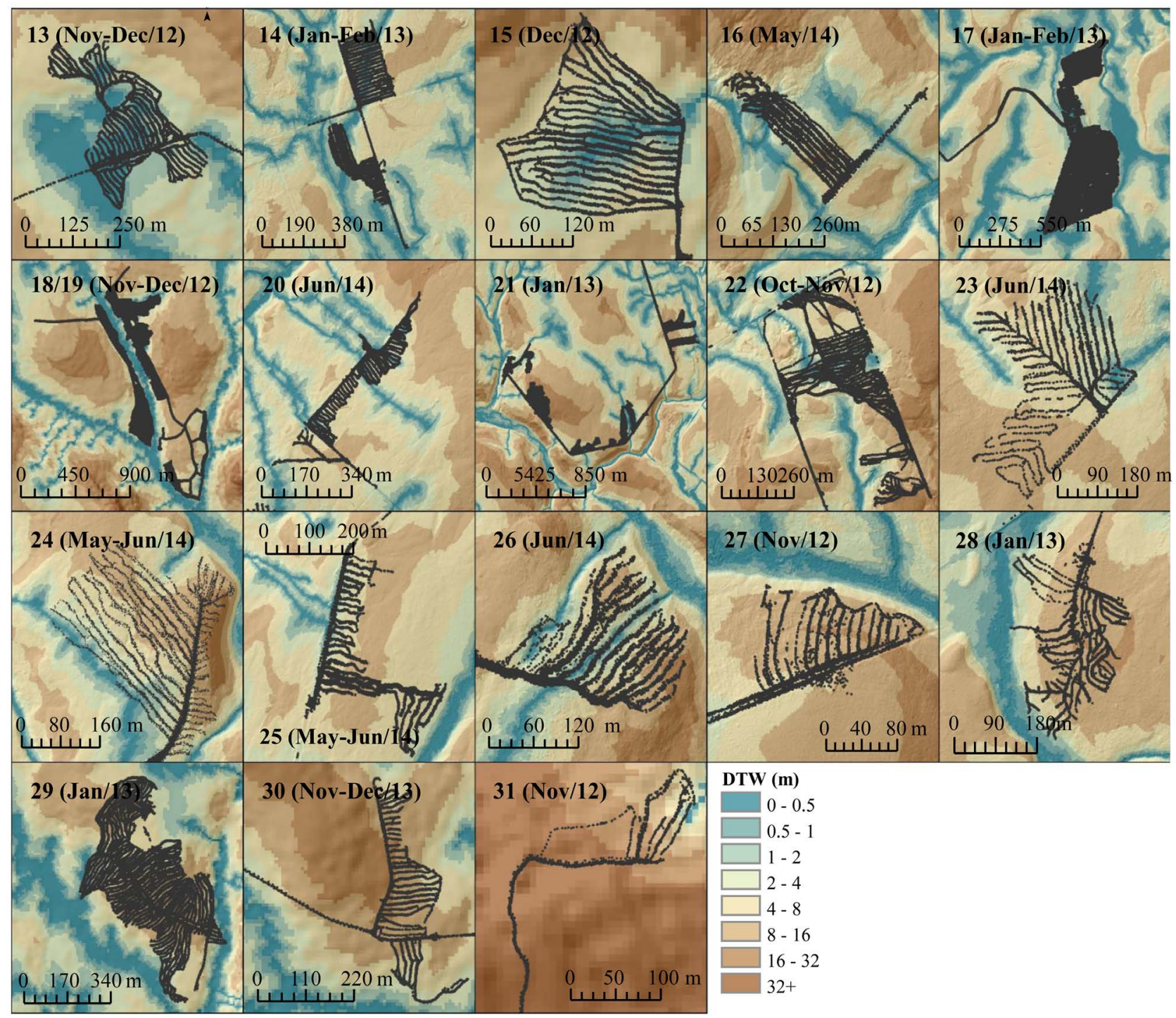

Figure 7. Wood-forwarding track patterns within JD15 harvest blocks, showing clearance sensor points overlaid on the DEM-derived DTW patterns, based on 4 ha upslope flow-initiation areas.

\subsection{Normalized Clearance Distribution Patterns}

The histograms of the standardized clearances per block followed a left-skewed pattern, as shown in Appendix 1 (Figure A1-1, Figure A1-2, and Figure A1-3). The corresponding Equation (1) generated probability distributions were overlaid on these histograms, with corresponding best-fitted $k, c_{\max }$ and $\theta$ values listed in Table 4, by harvest block. Examining these values revealed that $\log _{10} k$ correlates with $\theta$ such that

$$
\log _{10} k=(1.16 \pm 0.05)-(0.76 \pm 0.09) \theta \quad R^{2}=0.602
$$

In addition, the combination of $k$ and $\theta$ as in $\log _{10} k \theta$ correlates with $c_{\max }$ and machine type, i.e.

$$
\log _{10} k \theta=-(1.7 \pm 0.2)-(1.7 \pm 0.2) c_{\max }+(0.22 \pm 0.02) \mathrm{JD} 11 R^{2}=0.755
$$

(JD11, JD15 and TC coded 1 when present, otherwise 0 ). 


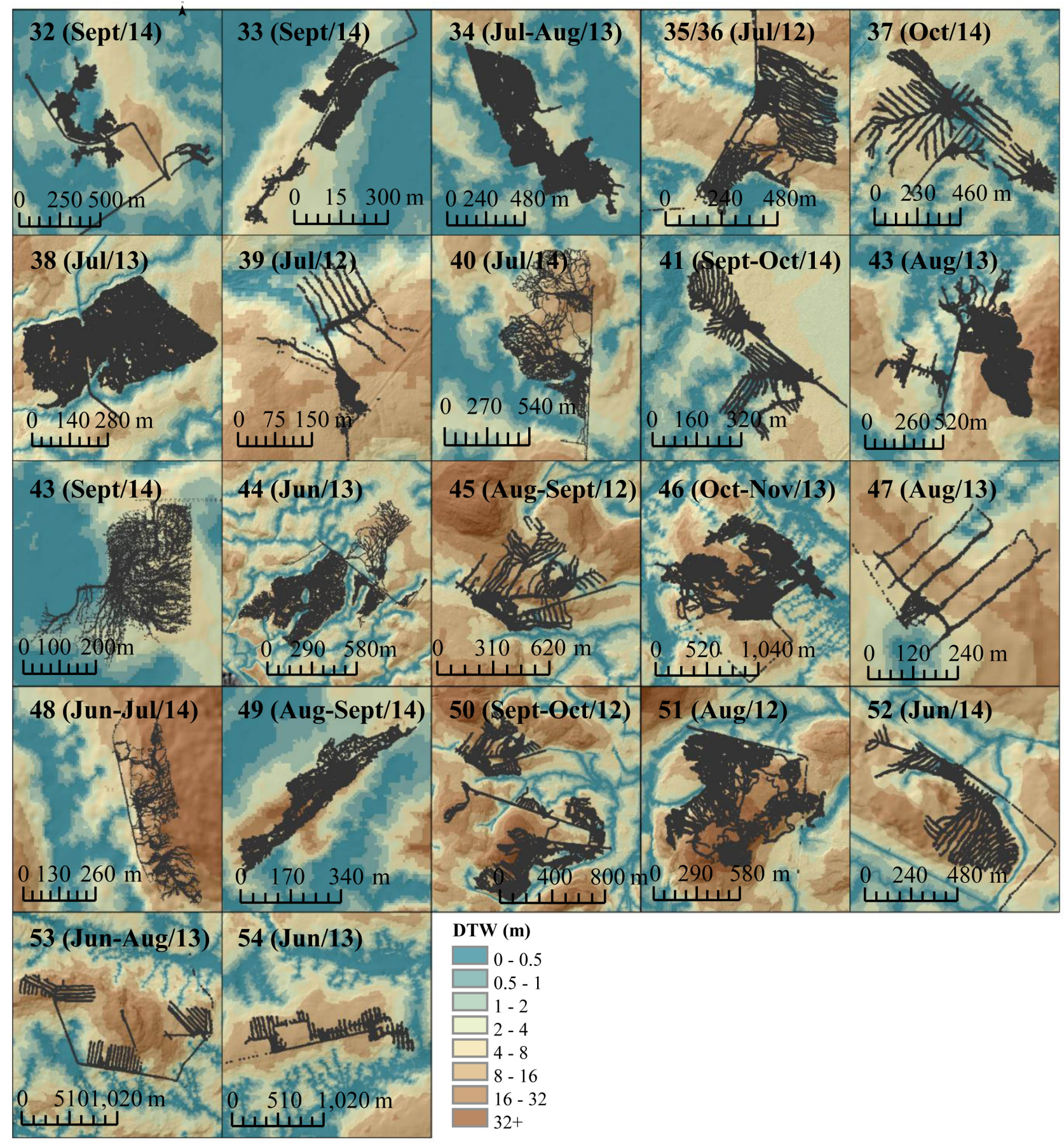

Figure 8. Wood-forwarding track patterns within TC harvest blocks, showing clearance sensor points overlaid on the DEM-derived DTW patterns, based on 4 ha upslope flow-initiation areas.

In general, increasing $k \theta$ values reflect a broadening of the clearance frequency distributions, and Equation (3) implies that this broadening increases with increasing $c_{\max }$, with further increases observed for the JD11 operations. This can be attributed to differences in machine operation, as follows:

1) JD11 was mainly used for commercial thinning, which involved generating brushmats from tree delimbing and topping by single-grip harvesters along 


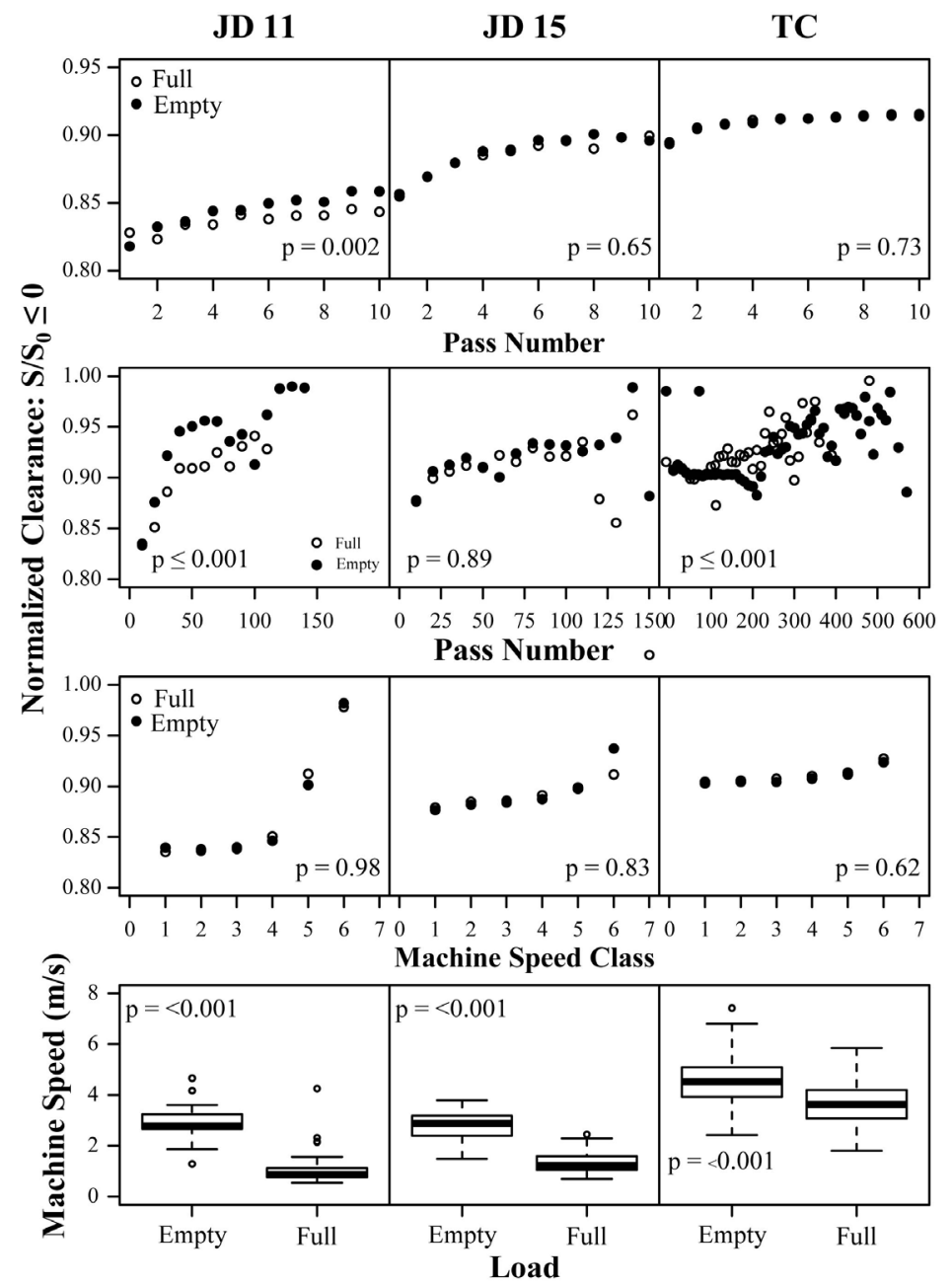

Figure 9. Mean machine specific normalized clearance versus number of passes (first 10 and all passes), empty versus full loads, and machine speed versus empty or full loads.

Table 3. Variability in normalized clearance, number of passes, speed by machine and operation type.

\begin{tabular}{ccccccccc}
\hline & & \multicolumn{2}{c}{ JD11 } & \multicolumn{2}{c}{ JD15 } & \multicolumn{3}{c}{ TC } \\
\cline { 3 - 9 } & & CT & CC & CT & SHW & CC & CT & SHW \\
\hline \multirow{3}{*}{ Normalized } & Mean & 0.84 & 0.88 & 0.89 & 0.89 & 0.91 & 0.92 & 0.91 \\
Clearance & Min & 0.11 & 0.1 & 0.1 & 0.1 & 0.08 & 0.08 & 0.09 \\
& Max & 1 & 1 & 1 & 1 & 1 & 1 & 1 \\
& Mean & 7 & 11 & 12 & 8 & 28 & 10 & 29 \\
Number of & SD & 12 & 19 & 18 & 14 & 39 & 10 & 40 \\
Passes (n) & Min & 1 & 1 & 1 & 1 & 1 & 1 & 1 \\
& Max & 252 & 186 & 198 & 154 & 548 & 58 & 568 \\
& Mean & 1.7 & 2.14 & 2.17 & 1.92 & 3.96 & 4.03 & 4.7 \\
& SD & 2.73 & 2.8 & 2.68 & 2.56 & 3.63 & 4.24 & 4.11 \\
Speed (m/s) & Min & 0 & 0 & 0 & 0 & 0 & 0 & 0 \\
& Max & 24 & 24 & 23.17 & 23.17 & 24 & 23.65 & 23.98 \\
\hline
\end{tabular}


Table 4. Best-fitted Equation (1) and Equation (5) regression results for the clearance frequency distributions and normalized clearances $\leq 1$, by machine and block; speed, passes, and DTW coefficients $\left(\times 10^{-2}\right) ; \mathrm{f}=0.122 \pm 0.002$.

\begin{tabular}{|c|c|c|c|c|c|c|c|c|c|c|c|}
\hline \multirow{3}{*}{ Block Id ${ }^{1}$} & \multirow{3}{*}{ n } & \multirow{3}{*}{$\begin{array}{c}\text { 10th } \\
\text { percentile }\end{array}$} & \multirow{2}{*}{\multicolumn{4}{|c|}{$\begin{array}{c}\text { Gamma Distribution Function (Equation (1)) } \\
\text { Parameters (+SE) }\end{array}$}} & \multicolumn{5}{|c|}{ Normalized Clearances $\leq 1$ (Equation (5)) } \\
\hline & & & & & & & \multirow{2}{*}{$\begin{array}{l}\text { Intercept } \\
\quad( \pm S E)\end{array}$} & \multicolumn{3}{|c|}{ Coefficients $( \pm \mathrm{SE})$} & \multirow{2}{*}{ Adj. $R^{2}$} \\
\hline & & & $\gamma(\mathrm{Bs})$ & $\mu(B x)$ & $\beta(k)$ & (A). K & & Speed & Passes & DTW & \\
\hline 2 & 284 & 0.64 & $0.47(0.08)$ & $1.22(0.05)$ & $10.01(2.94)$ & 0.99 & $0.69(0.02)$ & $1.09(0.21)$ & $10.95(1.72)$ & $0.51(0.22)$ & 0.33 \\
\hline 3 & 200 & 0.72 & $0.56(0.08)$ & $1.04(0.02)$ & $3.45(0.75)$ & 0.99 & $0.81(0.07)$ & $2.34(0.15)$ & & $0.69(0.15)$ & 0.59 \\
\hline 4 & 1750 & 0.66 & $0.53(0.01)$ & $1.13(0.03)$ & $6.58(1.99)$ & 0.99 & $0.72(0.01)$ & $1.68(0.07)$ & $10.35(0.07)$ & & 0.4 \\
\hline 7 & 1680 & 0.65 & $0.65(0.05)$ & $1.21(0.03)$ & $6.63(0.91)$ & 0.99 & $0.69(0.01)$ & $1.69(0.11)$ & $12.00(0.81)$ & $-0.53(0.14)$ & 0.21 \\
\hline 8 & 1047 & 0.65 & $0.50(0.13)$ & $1.19(0.60)$ & $8.57(3.54)$ & 0.99 & $0.70(0.01)$ & $1.61(0.12)$ & $13.90(0.66)$ & $-0.91(0.12)$ & 0.4 \\
\hline 9 & 366 & 0.63 & $0.50(0.01)$ & $1.19(0.05)$ & $9.06(3.13)$ & 0.99 & $0.71(0.02)$ & $1.04(0.17)$ & $19.77(1.17)$ & $-1.31(0.32)$ & 0.48 \\
\hline 10 & 996 & 0.65 & $0.44(0.14)$ & $1.22(0.05)$ & $10.82(5.09)$ & 0.99 & $0.69(0.01)$ & $1.65(0.09)$ & $12.94(0.58)$ & $-0.20(0.09)$ & 0.52 \\
\hline 11 & 1038 & 0.64 & $0.64(0.12)$ & $1.15(0.03)$ & $6.00(1.46)$ & 0.98 & $0.72(0.01)$ & $1.84(0.10)$ & $8.74(0.45)$ & & 0.42 \\
\hline 12 & 124 & 0.77 & $0.23(0.01)$ & $1.20(0.01)$ & $12.44(0.79)$ & 0.81 & $0.85(0.02)$ & & & $1.33(0.51)$ & 0.04 \\
\hline 13 & 242 & 0.78 & $0.60(0.04)$ & $1.30(0.02)$ & $6.73(0.68)$ & 0.99 & $0.77(0.02)$ & & $7.23(1.04)$ & $1.07(0.27)$ & 0.19 \\
\hline 14 & 989 & 0.84 & $0.43(0.01)$ & $1.30(0.01)$ & $8.01(0.01)$ & 0.98 & $0.87(0.01)$ & & $4.45(0.62)$ & & 0.05 \\
\hline 15 & 210 & 0.78 & $0.77(0.12)$ & $1.17(0.02)$ & $3.14(0.62)$ & 0.94 & $0.79(0.02)$ & $1.23(0.20)$ & $5.62(0.96)$ & & 0.25 \\
\hline 16 & 326 & 0.77 & $0.54(0.04)$ & $1.17(0.01)$ & $5.04(0.60)$ & 0.91 & $0.83(0.02)$ & $0.60(0.19)$ & $3.57(1.32)$ & & 0.04 \\
\hline 17 & 2550 & 0.81 & $0.57(0.08)$ & $1.25(0.03)$ & $5.36(1.15)$ & 0.99 & $0.83(0.01)$ & & $5.61(0.36)$ & $0.18(0.08)$ & 0.09 \\
\hline 18 & 96 & 0.78 & $0.71(0.04)$ & $1.25(0.01)$ & $4.44(0.22)$ & 0.94 & $0.94(0.04)$ & & $-6.52(2.96)$ & & 0.04 \\
\hline 19 & 134 & 0.82 & $0.56(0.02)$ & $1.26(0.01)$ & $5.45(0.21)$ & 0.93 & $0.82(0.01)$ & $0.61(0.21)$ & $4.10(1.22)$ & $0.42(0.19)$ & 0.18 \\
\hline 20 & 78 & 0.87 & $0.16(0.01)$ & $1.18(0.01)$ & $13.64(0.41)$ & 0.98 & $0.79(0.03)$ & $0.01(0.01)$ & & $2.18(0.49)$ & 0.27 \\
\hline 21 & 674 & 0.8 & $0.94(0.09)$ & $1.16(0.01)$ & $2.36(0.25)$ & 0.89 & $0.78(0.01)$ & $0.60(0.12)$ & $8.53(0.75)$ & & 0.17 \\
\hline 23 & 219 & 0.84 & $0.30(0.05)$ & $1.21(0.02)$ & $9.06(2.08)$ & 0.97 & $0.79(0.02)$ & & $12.15(1.65)$ & & 0.2 \\
\hline 24 & 2146 & 0.77 & $0.49(0.07)$ & $1.14(0.01)$ & $4.81(0.84)$ & 0.98 & $0.92(0.01)$ & $0.30(0.01)$ & & $-0.79(0.11)$ & 0.03 \\
\hline 25 & 357 & 0.82 & $0.37(0.06)$ & $1.16(0.02)$ & $6.71(1.41)$ & 0.99 & $0.97(0.02)$ & $0.57(0.17)$ & $-2.87(1.00)$ & $-0.91(0.34)$ & 0.08 \\
\hline 26 & 807 & 0.79 & $0.35(0.04)$ & $1.22(0.01)$ & $9.46(0.38)$ & 0.99 & $0.84(0.01)$ & $0.50(0.13)$ & $1.51(0.61)$ & $0.47(0.12)$ & 0.05 \\
\hline 28 & 382 & 0.85 & $0.76(0.04)$ & $1.26(0.01)$ & $3.85(0.33)$ & 0.86 & $0.80(0.01)$ & & $7.26(0.86)$ & & 0.16 \\
\hline 29 & 275 & 0.82 & $0.66(0.11)$ & $1.22(0.01)$ & $4.24(0.45)$ & 0.99 & $0.82(0.01)$ & & $4.88(0.70)$ & $0.39(0.18)$ & 0.19 \\
\hline 30 & 56 & 0.76 & $0.98(0.01)$ & $1.16(0.01)$ & $2.51(0.29)$ & 0.97 & $0.60(0.04)$ & $1.10(0.38)$ & $13.10(3.40)$ & $2.12(0.48)$ & 0.53 \\
\hline 31 & 32 & 0.89 & $0.30(0.01)$ & $1.20(0.01)$ & $7.05(0.27)$ & 0.97 & $0.71(0.03)$ & & $7.83(3.20)$ & $2.02(0.36)$ & 0.66 \\
\hline 32 & 496 & 0.71 & $0.97(0.05)$ & $1.17(0.01)$ & $3.30(0.01)$ & 0.95 & $0.87(0.01)$ & $-0.36(0.15)$ & $-6.86(0.71)$ & $2.31(0.19)$ & 0.4 \\
\hline 33 & 282 & 0.74 & $0.58(0.04)$ & $1.26(0.01)$ & $6.56(0.43)$ & 0.94 & $0.77(0.02)$ & $0.43(0.21)$ & $-3.70(0.87)$ & $3.64(0.35)$ & 0.3 \\
\hline 34 & 306 & 0.87 & $0.40(0.03)$ & $1.19(0.01)$ & $5.25(0.73)$ & 0.98 & $0.96(0.01)$ & & & $-1.41(0.21)$ & 0.13 \\
\hline 35 & 1300 & 0.7 & $0.51(0.06)$ & $1.16(0.01)$ & $4.33(0.76)$ & 0.94 & $0.80(0.01)$ & $0.60(0.07)$ & $6.78(0.36)$ & & 0.25 \\
\hline 36 & 1880 & 0.85 & $0.28(0.04)$ & $1.23(0.02)$ & $9.95(2.08)$ & 0.94 & $0.87(0.01)$ & $0.30(0.07)$ & $3.16(0.27)$ & & 0.08 \\
\hline 37 & 2264 & 0.79 & $0.47(0.03)$ & $1.20(0.01)$ & $6.05(0.63)$ & 0.99 & $0.94(0.01)$ & & $-5.06(0.34)$ & $0.40(0.11)$ & 0.09 \\
\hline 38 & 2881 & 0.85 & $0.38(0.05)$ & $1.27(0.02)$ & $8.18(1.65)$ & 0.98 & $0.83(0.01)$ & $0.17(0.06)$ & $1.51(0.24)$ & $1.21(0.05)$ & 0.17 \\
\hline 39 & 586 & 0.92 & $0.21(0.03)$ & $1.21(0.01)$ & $10.64(2.16)$ & 0.99 & $0.88(0.02)$ & $0.23(0.15)$ & & $0.81(0.30)$ & 0.01 \\
\hline
\end{tabular}




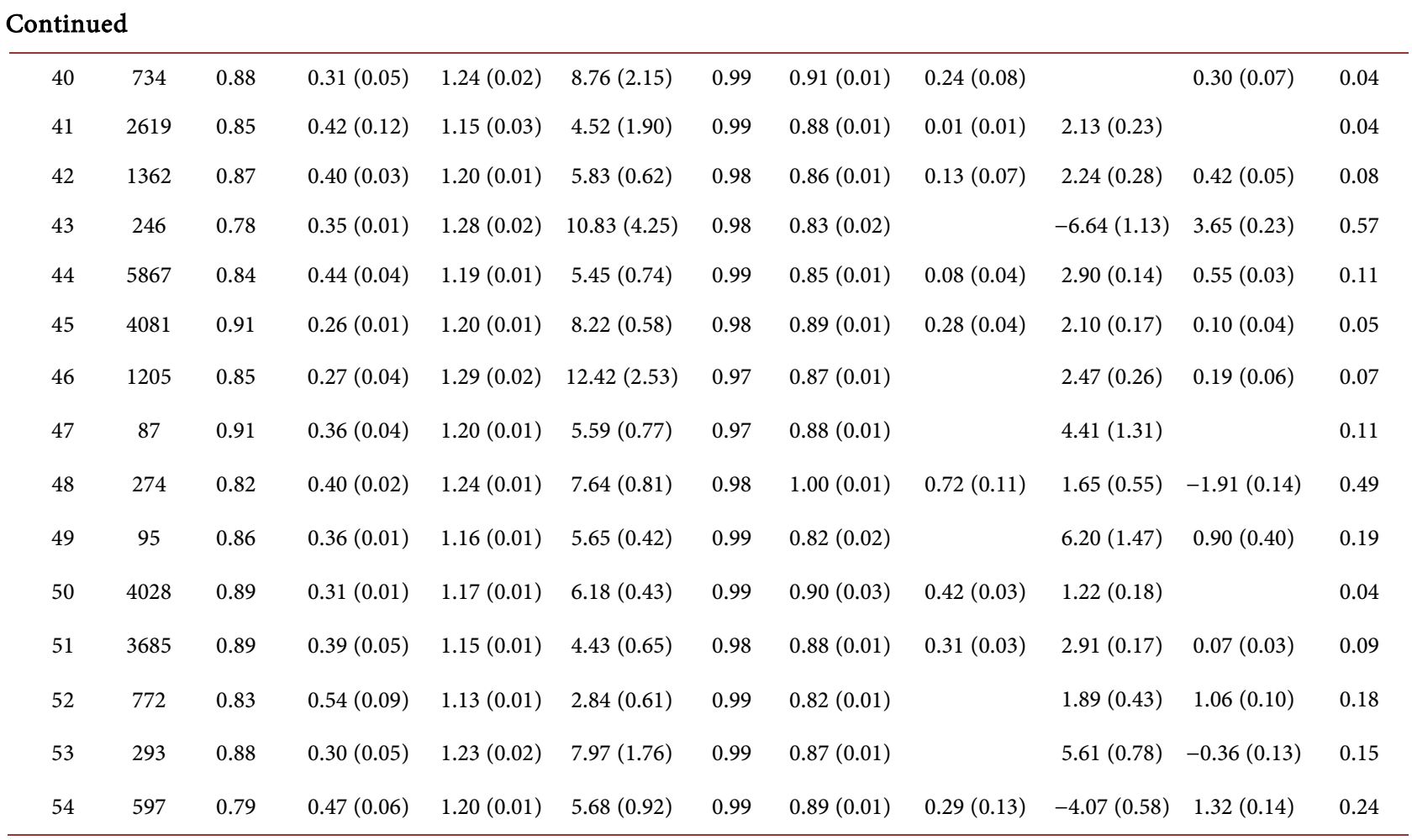

${ }^{1}$ Blocks $1,5,6,22$, and 27 had missing sensor data and were therefore removed from the analysis.

wood forwarding trails; the broadening of the clearance distributions would be due to the ultrasonic signals bouncing off machine-induced brush-mat sagging and lifting.

2) By machine type, the $k \theta$ product varied as follows: JD11 $=3.9 \pm 0.4$, JD15 $=$ $2.8 \pm 0.1 ; \mathrm{TC}=2.5 \pm 0.1$; i.e., JD11 significantly higher than JD15 and TC ( $\mathrm{p}$-value $<0.001$ ), and JD15 is significantly higher than TC ( $\mathrm{p}$-value $=0.14$ ).

3) Using the $<10 \%$ tail of the standardized clearance marker produced the following sequence: JD11 $=0.67 \pm 0.02$; JD15 $=0.80 \pm 0.02$, $\mathrm{TC}=0.834 \pm 0.01$ (p-value $<0.05$ ). Hence, forwarding wood on brushmats produced longer standardized clearance trails towards zero-clearance than forwarding on bare ground. By Equation 4, the $10^{\text {th }}$ percentile clearances per block are directly relatable to the best-fitted Gamma distribution parameters as follows:

$$
\begin{aligned}
& 10^{\text {th }} \text { percentile clearances } \\
& =-(0.35 \pm 0.10)-(0.73 \pm 0.4) \log _{10} k \theta+(1.23 \pm 0.09) c_{\max } \quad R^{2}=0.892
\end{aligned}
$$

4) Trails for the shelterwood and clear-cut operations without brushmats had standardized clearance peaks at or near 1 . Clearances greater than 1 were due to machine movements over stumps, rocks and uneven ground. The $c_{\max }$ ranges were similar by machine type, as follows: $1.04<$ JD11 < 1.22; $1.14<$ JD15 $<1.30$; $1.13<\mathrm{TC}<1.29$.

\subsection{Box Plots}

The normalized data with clearances $<1$ are presented in Appendix 2 by way of 
box plots showing $10^{\text {th }}, 25^{\text {th }}, 50^{\text {th }}, 75^{\text {th }}$ and $90^{\text {th }}$ normalized clearance percentiles and associated outliers per block and machine type by number of passes, speed and DTW classes (Figure A2-1, Figure A2-2, and Figure A2-3). These plots show that machine clearances varied from block to block in relation by machine operations. For TC, these variations were in part attributable to the variable June to November cumulative precipitation pattern from 2012 to 2014 (Figure 10), with lower clearances less prominent in the fall of 2012 following dry summer conditions, but more prominent in summer blocks where DTW $<1 \mathrm{~m}$ (Blocks 32, 35, 36; Figure A2-3).

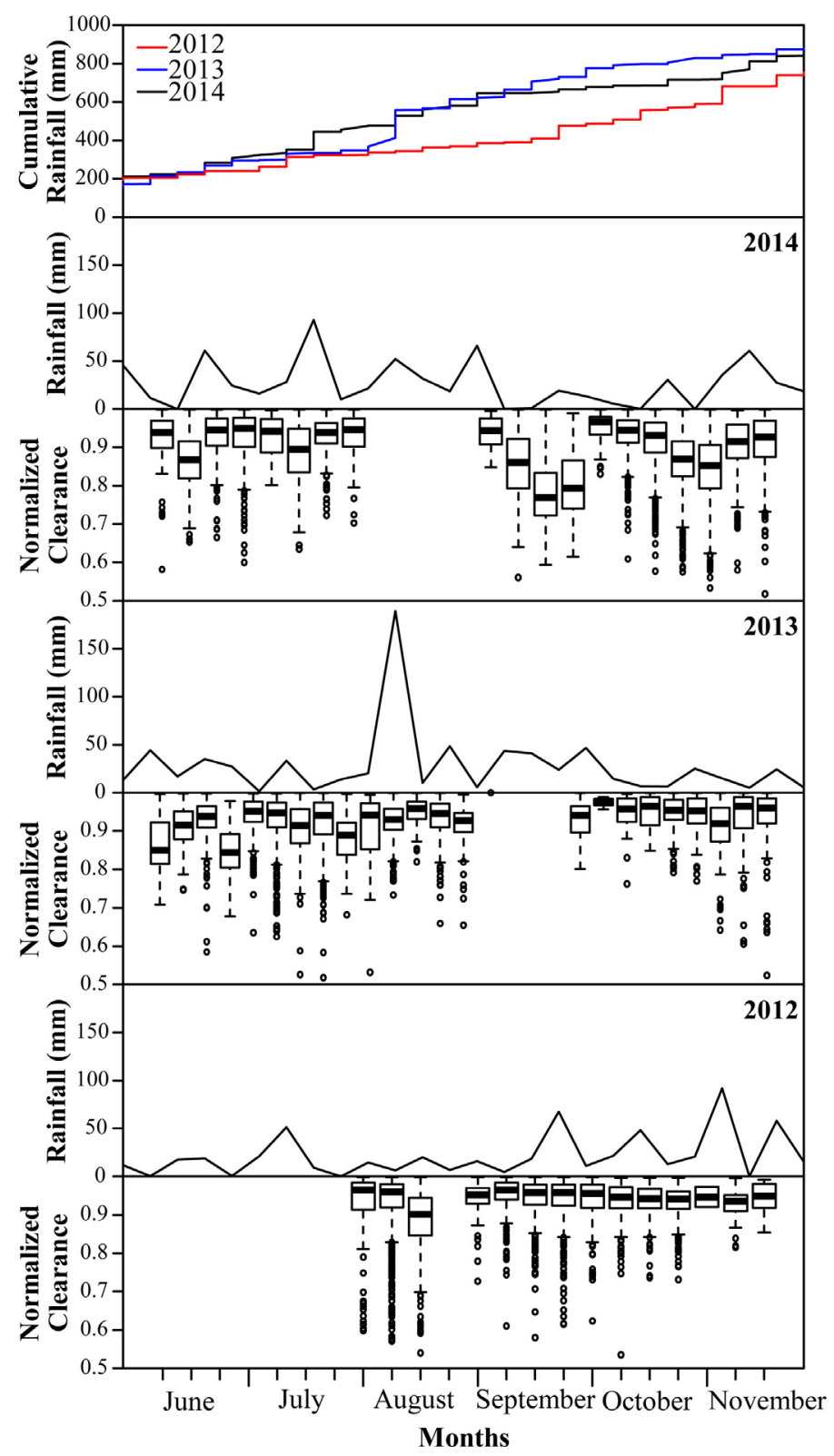

Figure 10. Weekly and cumulative May to December rainfall for 2012-2014 (top), also showing 10th, 25th, 50th, 75th and 90th percentile box plots for the normalized TC sensor clearances $\leq 1$. 


\subsection{Normalized Clearance $\leq 1$}

Regressing the normalized clearances $\leq 1$ values against number of passes, speed and DTW classes by way of

normalized clearance $\leq 1=$ intercept $+k_{\text {pass }}$ passes $+k_{\text {speed }}$ speed $+k_{\text {DTW }} \mathrm{DTW}$

produced the best-fitted interception and regression coefficient results also listed in Table 4, with $\mathrm{R}^{2}$ values ranging from near 0 to 0.67 . These results are summarized as follows:

1) Normalized clearances increased with increasing machine speed, with only one reduction registered on a wet TC trail (block \# 32).

2) Increasing the number of wood-forwarding passes per track had mostly positive to no clearance effects, with blocks \# 18 and \#25 being exceptions. For the TC wood-skidding operations, the influence of pass numbers per track on clearances varied from mainly positive during dry conditions to negative during wet conditions.

3) The influence of DTW on TC clearances also varied from positive to negative. The positive trend occurred when the ground was dry due to increased soil resistance to compaction. The negative trend occurred when operating on wet ground during and after soil-saturating rain events, due to decreased soil resistance to compaction and tire slippage.

Analyzing the normalized clearance intercepts in Table 4 in terms of their associated speed, number of passes and DTW regression coefficients generated the following multiple regression result:

$$
\begin{aligned}
& \text { Normalized clearance intercept } \\
& =(0.910 \pm 0.04)-(1.23 \pm 0.06) k_{\text {pass }}-(0.041 \pm 0.005) k_{\text {speed }} \\
& -(0.052 \pm 0.003) k_{\text {DTw }} ; \quad R^{2}=0.946 ; \text { RMSE }=0.020
\end{aligned}
$$

This equation implies that the normalized clearance intercept:

1 ) is equal to $0.910 \pm 0.04$, or $75 \%$ to $78 \%$ actual machine-to-ground clearances on average, when machine speed, number of passes and DTW have no clearance influence; in terms of actual depth, this number amounts to about 12 $\mathrm{cm}$; a considerable part of this would be due to the compression of the organic forest floor accumulations;

2) decreases below 0.910 on dry to moist and wet soils with increasing number of passes and/or machine speed, this would be due to repeating soil compaction along the same track and increased shearing stress with increased tire rotation;

3) decreases below 0.910 as the DTW-projected influence on soil resistance to compaction becomes stronger from very poor (where DTW is near 0) to well and excessive (where DTW is $>1 \mathrm{~m}$ ); i.e., a positive DTW influence causes the regression intercept to be lowest at DTW $=0$; a negative DTW influence would do the opposite and may occur where DTW $>0$ due to increased slope-induced wheel slippage.

Equation (6) suggests that the lowest normalized intercept for class-averaged normal clearances $\leq 1$ would amount to $0.910-1.23 \times\left(k_{\text {pass }}=0.19\right)-0.041 \times$ 
$\left(k_{\text {speed }}=2.34\right)-0.052 \times\left(k_{\mathrm{DTW}}=3.65\right)=0.39$. The lowest average normalized clearance intercept per number of pass, speed and DTW classes registered in Table 4 was 0.60 , for block \# 30 (JD15, Nov. 2012). This is approximately equal to chassis clearance, and this occurred on a well-drained soil (DTW $>2 \mathrm{~m}$ ) following a rain event. The lowest non-averaged normalized signal-to-ground surface distance was 0.43 , i.e., equivalent $60.1 \mathrm{~cm}$, thereby amounting to $19.5 \mathrm{~cm}$ below the reflecting ground surface. This occurred on block \# 15 in January 2013 when the ground was covered with snow.

The correlation matrix in Table 5 pertaining to the four Equation (6) variables indicates that the influence of number of passes and machine speed on the clearance intercepts were positively correlated with one another, while both were negatively correlated to DTW clearance influence. Hence, DTW-influenced softening of the ground tends to decrease machine speed and reduces repeat traffic along the same track.

\subsection{Block-Specific Examples}

An example of low to extensive rutting is shown in Figure 11 for TC block \# 41 (Sept.-Oct. 2014), where soil conditions varied from dry to moist to wet due to season- and weather-induced variations in local DTW patterns. Moderate soil compression occurred along trails where soils remained dry at DTW $>1 \mathrm{~m}$, with compression decreasing towards zero with increasing number of passes. On the landing site where DTW was $<0.5 \mathrm{~m}$, soil ruts were deeper and number of passes per track increased up to 270 . For the trail through transitional and somewhat sloped DTW zones, normalized clearances decreased with increasing number of passes per track. In general, the extent of soil compaction was highest at or near the plastic soil moisture limits, while soil displacement was highest at and above the liquid soil moisture limits.

The data for machine clearance, number of passes per track and speed are illustrated in Figure 12 for harvest blocks 10, 32, 33, 43. The mapped dots so shown demonstrate the following effects:

1) Normalised clearances along the central wood forwarding road in block 10 approached 1 at high track numbers and speed.

2) Rutting as indicated by persistently low clearances occurred along the most

Table 5. Correlation matrix (with p-values) for Equation (6) variables and associated block entries in Table 4.

\begin{tabular}{ccccc}
\hline & Speed & Number of passes & DTW & Intercept \\
\hline Speed & $1.00(0.00)$ & & & \\
Number of passes & $0.76(0.00)$ & $1.00(0.00)$ & & \\
DTW & $-0.34(0.11)$ & $-0.45(0.02)$ & $1.00(0.00)$ & \\
Intercept & $-0.62(0.00)$ & $-0.75(0.00)$ & $-0.29(0.09)$ & $1.00(0.00)$ \\
\hline $\mathrm{n}=49$. & & & & \\
\end{tabular}




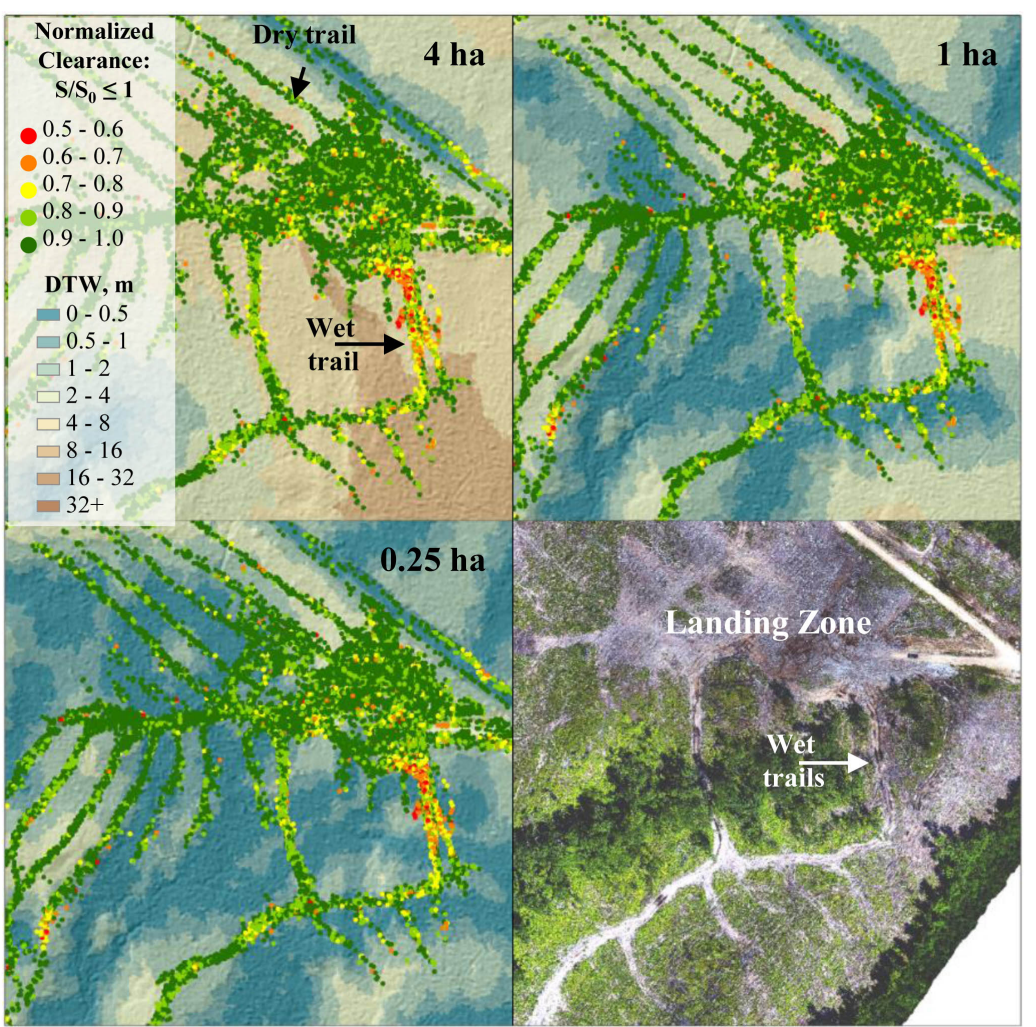

(a)

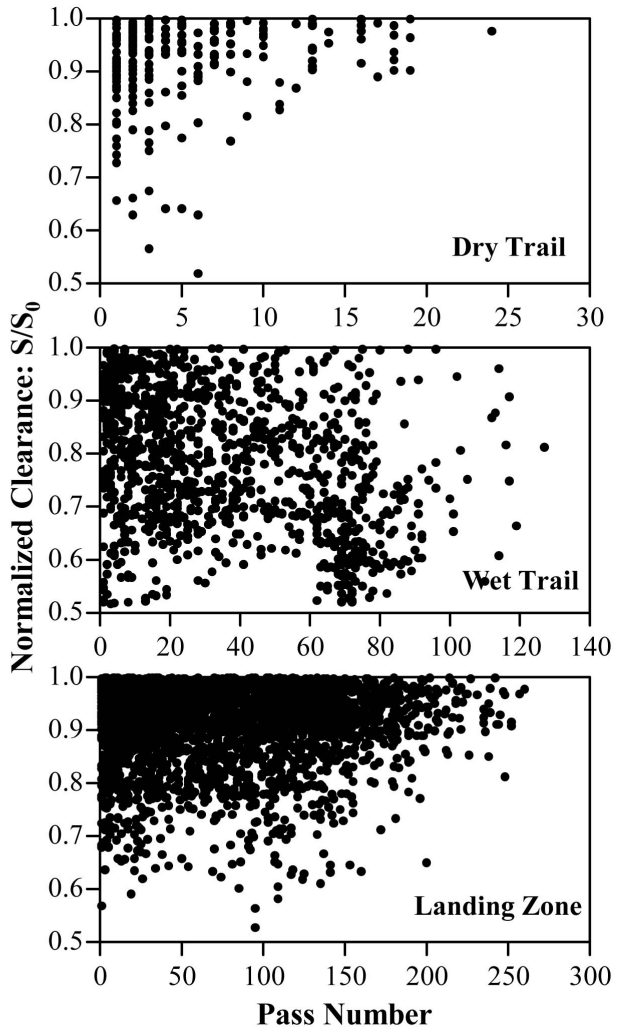

(b)

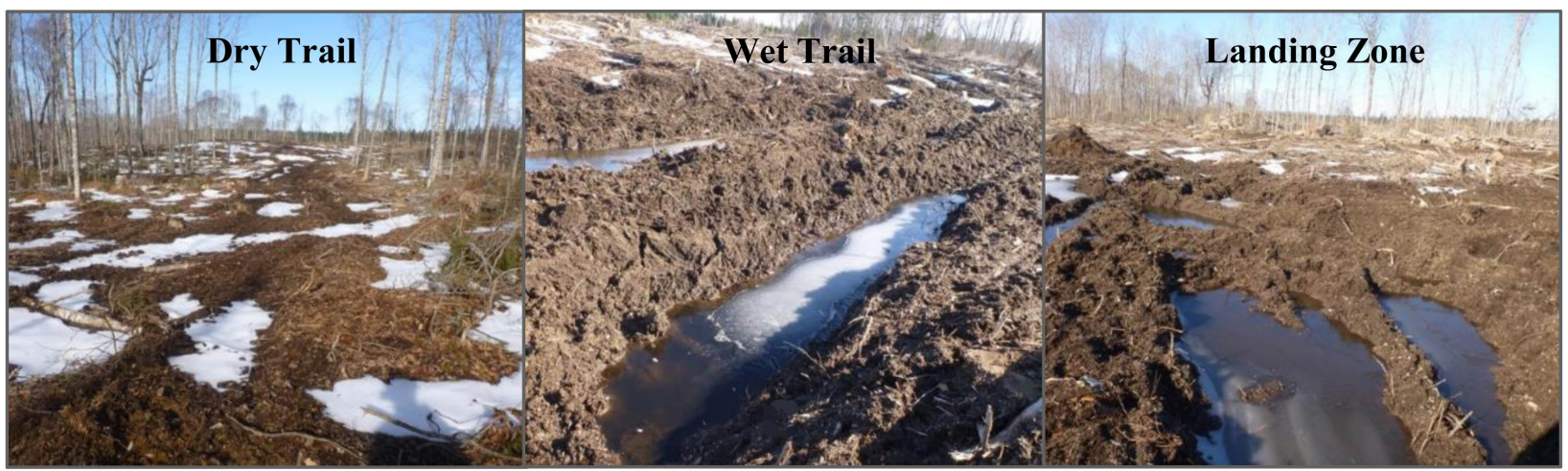

(c)

Figure 11. (a): TC block \#41 showing weather-affected DEM-derived DTW patterns based on assuming 4 ha (top-left), 1 ha (top-right) and 0.25 ha (bottom-left) of upslope flow accumulation area for channel-flow initiation. The high-resolution surface image (bottom-right) shows ruts with low machine clearances along high multiple-pass connector trails; (b): block 41 normalized clearance versus increasing number of passes along dry and wet forwarding trails and on landing site; (c): dry trail (no rutting), wet trail (deep rutting), and landing site (braided rutting) images. Normalized clearance in terms of chassis- to sensor-to solid-ground distances (front): $\mathrm{M} 0 / \mathrm{S} 0=0.63$.

frequently used TC skidding passes as shown for blocks 43, 32, and 33; elsewhere, TC clearances tend to be near 1 . In contrast, the JD11 clearances along brush-matted wood forwarding tracks were lower due to brush-mat flexing.

3) TC machine track speeds varied more than JD11 track speeds, with TC being faster on higher DTW ground. JD11 speeds were consistently slower along the commercial wood forwarding tracks. 


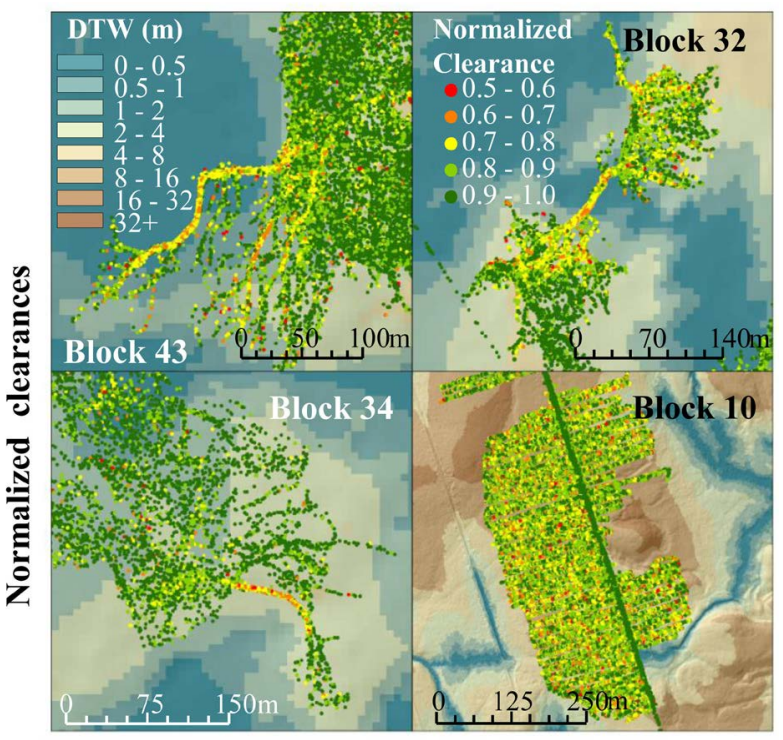

(a)

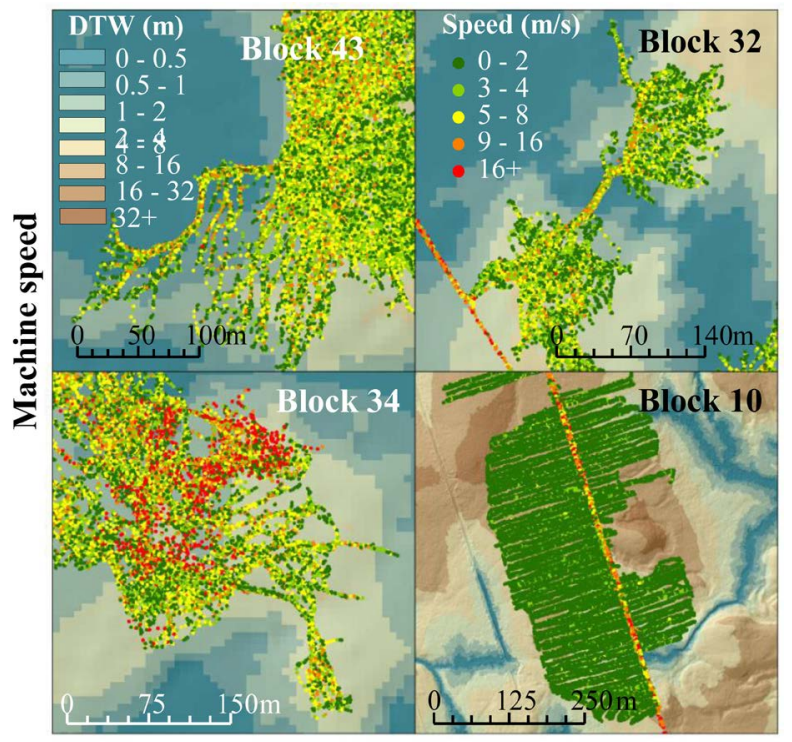

(c)

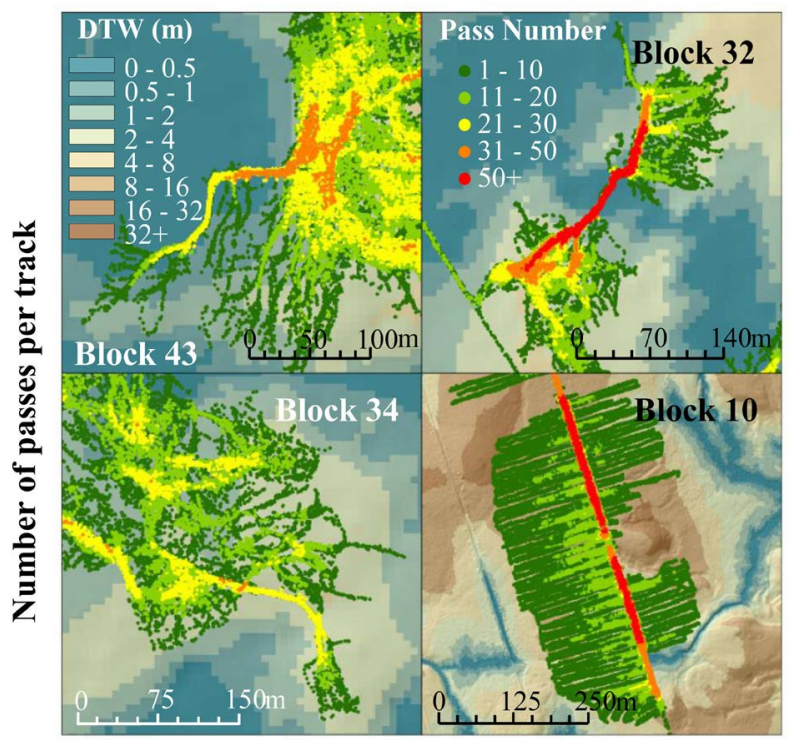

(b)

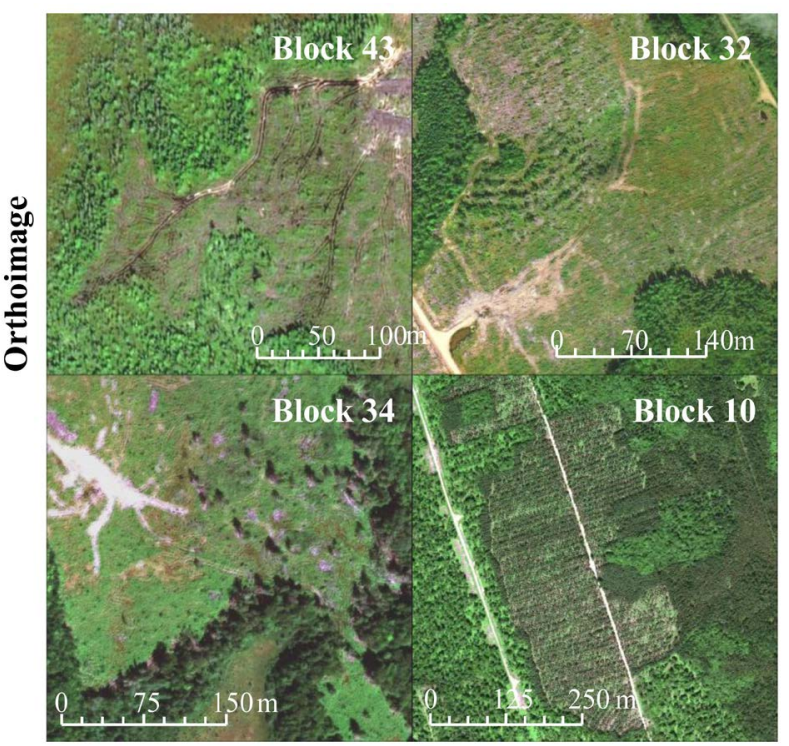

(d)

Figure 12. Visual correlation between DTW and normalized clearance (a), number of passes per track (b); and machine speed (c); for blocks 47, 32, 34, and 10. Ortho imagery of blocks (d) showcasing harvest type and soil disturbance (Imagery Source: Esri, DigitalGlobe, GeoEye, Earthstar Geographics, CNES/Airbus DS, USDA, USGS, AEX, Getmapping, Aerogrid, IGN, IGP, swisstopo, and the GIS User Community).

\subsection{Further Observations}

\subsubsection{Clearances by Machine Type and Number of Passes}

Some of the normalized clearance differences would be caused by differences in machine weight, loads, tire pressure, number of tires, and whether the tires were chained or tracked (Table 1). For example, TC had a higher front footprint pressure about 2 to 3 times higher than the JD11 and JD15 forwarders. Henceaccording to the Table 1 entries-soil compression should have been deeper with TC than with JD11 and JD15 operations. This was indeed the case along 
repeated TC forwarding tracks on moist to wet ground (Figure 12). Under dry conditions, however, normalized clearance patterns peaked around one, except for the lower JD11 brush-mat clearances. Compared to JD15, JD11-exerted footprint pressure was about double (Table 1). Consequently, and consistent with the experimental machine-load and brush-mat observations by Labelle and Jaeger (2012), JD11brush-mat clearances were not only lower than for JD15 brush-mat clearances, but JD11 clearance were also significantly lower during forwarding than returning (Figure 9). In terms of increasing number of passes, brushmat clearances flattened through repeated crushing and consolidation.

\subsubsection{Machine Speed}

Machine speeds are affected by soil-tire interactions, including soil compaction and soil displacement (Shmulevich et al., 1998). In this regard, Liu et al. (2009) reported increasing soil displacement with increased tire rotation on dry ground. On soft ground, however, slow traffic increased soil compression and soil displacement (Grahn, 1991; Taghavifar \& Mardani, 2014). For the JD11, JD15, and TC operations, machine speed was definitely affected by machine load, being lower when fully loaded than when empty, with TC speeds significantly greater than JD11 and JD15 speeds (Figure 9). This effect was likely due to operating under more open than dense stand conditions, i.e., forwarding logs following clear-cutting and shelter-wood cutting versus logs following forest plantation thinning.

\subsubsection{Bare-Ground Operations}

When operating on bare ground during clear-cutting and shelter-wood harvesting, off-road clearances can also be expected to decrease with increasing footprint pressure and increasing number of passes due to increasing soil compression (Jamshidi et al., 2008; Vega-Nieva et al., 2009; Taghavifar \& Mardani, 2014) (Table 1). This, however, did not happen because of lateral sensor restrictions and track broadening as the soil continued to be compressed with increasing number of passes (Figure 2 and Figure 9). In contrast, clearances were low on wet ground and repeatedly so with increasing number of passes due to deep and recurring soil displacements as exemplified in Figure 11. In addition, soil rutting would become even deeper on wet slopes through load- and slope-induced tire and track slippage.

\subsubsection{Season and Weather Details}

With varying soil wetness by weather and across seasons, there is a general correspondence between TC clearances and ground conditions (Figure 10). For TC, lower clearances and deeper soil rutting were incurred within or across the harvest blocks on account of: 1) low DTW locations, 2) snow accumulations, 3) snow melt and prolonged rain events, and 4) low evapotranspiration (Raven et al., 1999; Ács et al., 2011; Jones \& Arp, 2017) before leaf-out (May-June) and after leaf-fall (October-November). In contrast, JD11 and JD15 clearances were 
less relatable to the varying ground conditions due to low footprint pressure (Table 1) and brush-mat operations.

An example of weather-induced soil rutting on dry ground $(4<\mathrm{DTW}<32 \mathrm{~m})$ is shown in the form of normalized clearances $<0.8$ in Figure 13. This occurred during TC operations in block \#48 two days after a $110 \mathrm{~mm}$ rain event in July 2014 (Hurricane Arthur). Similarly, Block \#41 operations immediately led to extensive soil rutting after a $30 \mathrm{~mm}$ rain event on wet October soils led to extensive soil rutting (Figure 11). To avoid such occurrences, wood forwarding operations were generally deferred to occur on dry ground during spring, summer and fall,

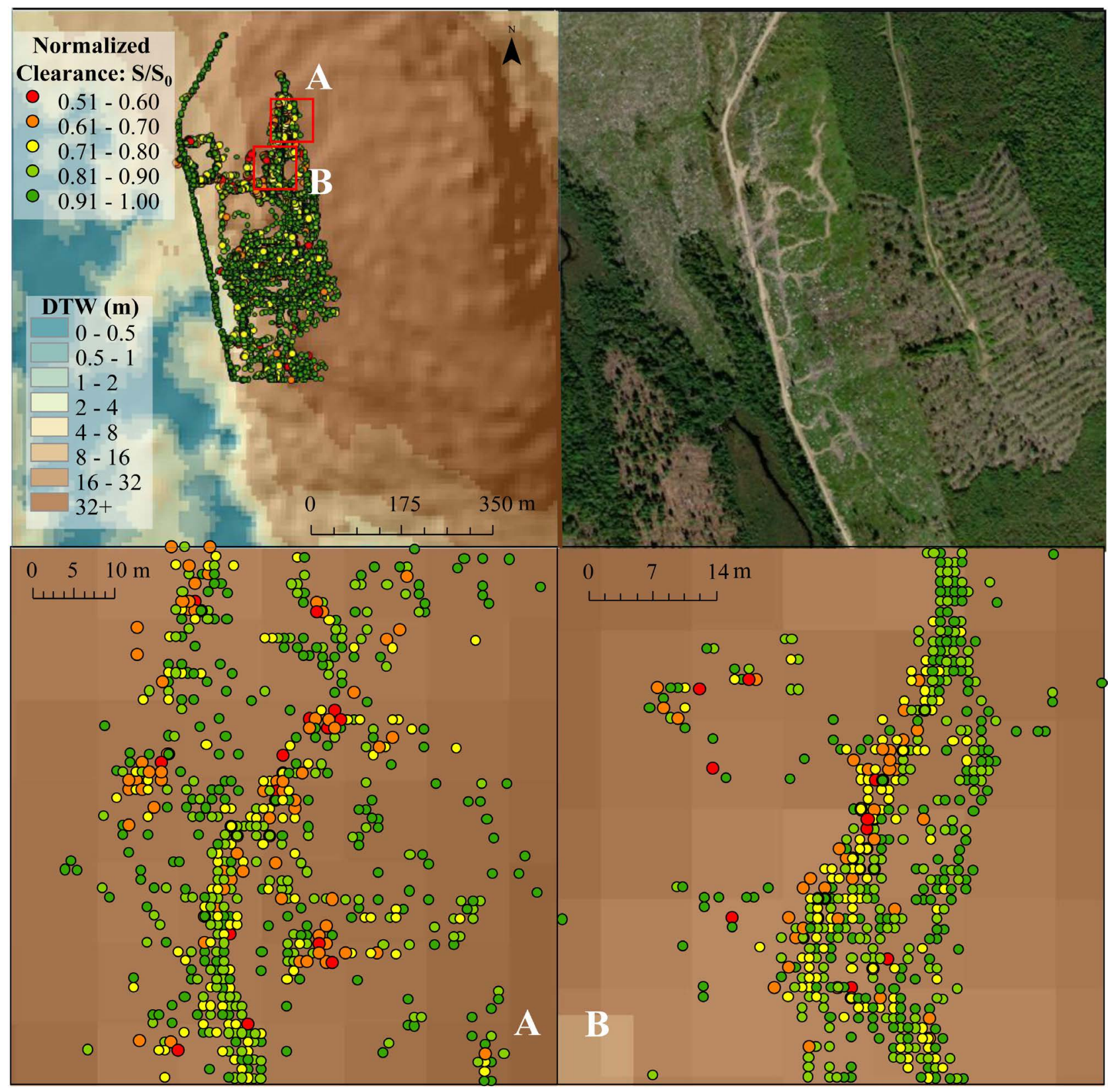

Figure 13. Normalized clearances across block 2, showing low TC clearances on DTW $>4 \mathrm{~m}$ ground following a $110 \mathrm{~mm}$ per day storm event in July 2014, with two close-ups (bottom panels; A, B red boxes in top panel). 
and on frozen ground during winter. During the JD11 and JD15 winter operations (block \#1, 12 - 15, 17 - 19, and 28 - 30), the normalized clearances were mostly due to ultrasonic reflections from snow surfaces. These clearances decreased with increasing number of passes due to snow compaction and track widening. This effect was most notable at NWU where the snowpack was almost twice as deep than at the southern locations (Figure A3-1). While snow compaction has no effect on frozen soil rutting, it has been shown to cause soils along the tracks to freeze deeper and longer, thereby delaying soil thawing along the established tracks (Grady, 1982; Garcia et al., 2015).

\section{Conclusions and Concluding Remarks}

The assessment of the machine clearance data provided the following insights:

1) The normalized clearances were affected by brushmat versus bareground operations, with the former producing lower but still broader clearance distributions than the latter.

2) All of the block-based clearance histograms followed asymmetric Gamma frequency distributions.

3) Clearances increased systematically with increasing passes towards 1 on dry ground due to successive soil compression and track widening. On wet ground, clearances would decrease because of successive soil re-displacements.

4) The TC centralizing wood-forwarding pattern following clear-cutting and shelter-wood operations included long tracks with number of passes exceeding 100 per track, which would lead to rutting along wet and wet-to-dry transitional ground conditions.

5) Number of passes, machine speed and low to high DTW classes all affected the normalized clearance results in terms of block and weather-specific conditions, with least effects registered along brushmat and dry-ground tracks, all based on fairly low machine footprint pressures in the order JD15 $<$ JD11 $<$ TC.

6) Clearances along the same bare-ground tracks do not necessarily reflect rut depths due to track broadening and using machine-fixed positions for machine-to-ground distance monitoring. On brushmats, the clearance data reflect brushmat re-conditioning due to repeated track traffic.

7) Actual machine clearance can be obstructed due to ultrasonic sensor blockage by way of debris, and by the presence of snowpack accumulations.

8) The extent of soil rutting versus machine clearance needs to be evaluated separately by way of, e.g., high-resolution block surveys following completion of block operations (Salmivaara et al., 2018), or through machine-mounted LiDAR-based ground scanning (Giannetti et al., 2017).

9) GPS tracking of tire rotation in connection with machine speed would assist in determining actual to potential soil displacement in terms of tire slippage and tread design.

\section{Acknowledgements}

This research was supported by J. D. Irving Limited (JDI) as a part of the 
NSERC-sponsored Cooperative Research Development (CRD) Project on Forest Soil Trafficability. Special thanks go Greg Adams at JDI for facilitating this project, and to FP Innovations personnel for the development and installations of the ultrasonic sensor data loggers. Also many thanks to the Shane Furze, Doug Hiltz, and John Paul Arp for help with data retrieval and GIS processing.

\section{References}

Ács, F., Breuer, H., \& Szász, G. (2011). Estimation of Actual Evapotranspiration and Soil Water Content in the Growing Season. Agrokémia és Talajt, 60, 57-74.

Balland, V., Pollacco, J. A. P., \& Arp, P. A. (2008). Modeling Soil Hydraulic Properties for a Wide Range of Soil Conditions. Ecological Modelling, 219, 300-316.

https://doi.org/10.1016/j.ecolmodel.2008.07.009

Berisso, F. E., Schjønning, P., Keller, T., Lamandé, M., Etana, A., de Jonge, L. W., Iversen, B. V., Arvidsson, J., \& Forkman, J. (2012). Persistent Effects of Subsoil Compaction on Pore size Distribution and Gas Transport in a Loamy Soil. Soil \& Tillage Research, 122, 42-51. https://doi.org/10.1016/j.still.2012.02.005

Brady, N. C., \& Weil, R. R. (2008). The Nature and Properties of Soils (14th ed.). Upper Saddle River, NJ: Prentice Hall.

Buja, K. (2012). Finding Overlapping Features Tool for ArcGIS. https://www.arcgis.com/home/item.html?id=968e6a55a11640d2b9cfa211104d3811

Czyz, E. A. (2004). Effects of Traffic on Soil Aeration, Bulk Density and Growth of Spring Barley. Soil \& Tillage Research, 79, 153-166. https://doi.org/10.1016/j.still.2004.07.004

Department of Environment and Climate Change Canada (2016). Historical Climate Data. http://climate.weather.gc.ca/

Devore, J. L. (1999). Probability and Statistics for Engineering and the Sciences (5th ed.). Pacific Grove, CA: Duxbury Press.

Ezzati, S., Najafi, A., Rab, M. A., \& Zenner, E. K. (2012). Recovery of Soil Bulk Density, Porosity and Rutting from Ground Skidding over a 20-Year Period after Timber Harvesting in Iran. Silva Fennica, 46, 521-538. https://doi.org/10.14214/sf.908

Garcia, M., Özdogan, M., \& Townsend, P. A. (2015). Impacts of Forest Harvest on Cold Season Land Surface Conditions and Land-Atmosphere Interactions in Northern Great Lakes States. Journal of Advances in Modeling Earth Systems, 6, 923-937. https://doi.org/10.1002/2014MS000317

GeoNB (2018). GIS Ecological Land Classification. Fredericton, New Brunswick: Department of Energy and Resource Development.

Giannetti, F., Chirici, G., Travaglini, D., Bottalico, F., Marchi, E., \& Cambi, M. (2017). Assessment of Soil Disturbance Caused by Forest Operations by Means of Portable Laser Scanner and Soil Physical Parameters. Soil Science Society of America Journal, 81, $1-23$.

Grady, T. R. (1982). The Effects of Snow Compaction on Water Release and Sediment Yield. MSc. Thesis, Bozeman, MT: Montana State University.

Grahn, M. (1991). Prediction of Sinkage and Rolling Resistance for Off-the-Road Vehicles Considering Penetration Velocity. Journal of Terramechanics, 28, 339-347. https://doi.org/10.1016/0022-4898(91)90015-X

Grigal, D. F. (2000). Effects of Extensive Forest Management on Soil Productivity. Forest Ecology and Management, 138, 167-185.

https://doi.org/10.1016/S0378-1127(00)00395-9 
Horn, R., Domżał, H., Słowińska-Jurkiewicz, A., \& van Ouwerkerk, C. (1995). Soil Compaction Processes and Their Effects on the Structure of Arable Soils and the Environment. Soil \& Tillage Research, 35, 23-36.

https://doi.org/10.1016/0167-1987(95)00479-C

Jamshidi, R., Jaeger, D., Raafatnia, N., \& Tabari, M. (2008). Influence of Two Ground-Based Skidding Systems on Soil Compaction under Different Slope and Gradient Conditions. International Journal of Forest Engineering, 19, 9-16.

Jones, M.-F., \& Arp, P. A. (2017). Relating the Cone Penetration and Rutting Resistance of Soils to Variations in Soil Properties and Daily Moisture Variations over Time. Open Journal of Soil Science, 7, 149-171. https://doi.org/10.4236/ojss.2017.77012

Kozlowski, T. T. (2008). Soil Compaction and Growth of Woody Plants. Scandinavian Journal of Forest Research, 14, 596-619. https://doi.org/10.1080/02827589908540825

Labelle, E. R., \& Jaeger, D. (2011). Soil Compaction Caused by Cut-to-Length Forest Operations and Possible Short-Term Natural Rehabilitation of Soil Density. Soil Science Society of America Journal, 75, 2314-2329. https://doi.org/10.2136/sssaj2011.0109

Labelle, E. R., \& Jaeger, D. (2012). Quantifying the Use of Brush Mats in Reducing Forwarder Peak Loads and Surface Contact Pressure. Croatian Journal of Forest Engineering, 33, 249-274.

Liu, K., Ayers, P. D., Howard, H., \& Anderson, A. (2009). Influence of Turning Radius on Wheeled Military Vehicle Induced Rut Formation. Journal of Terramechanics, 46, 49-55. https://doi.org/10.1016/j.jterra.2009.02.004

Murphy, P. N. C., Ogilvie, J., \& Arp, P. A. (2009). Topographic Modelling of Soil Moisture Conditions: A Comparison and Verification of Two Models. European Journal of Soil Science, 60, 94-109. https://doi.org/10.1111/j.1365-2389.2008.01094.x

Murphy, P. N. C., Ogilvie, J., Meng, F.-R., White, B., Bhatti, J. S., \& Arp, P. A. (2011). Modelling and Mapping Topographic Variations in Forest Soils at High Resolution: A Case Study. Ecological Modelling, 222, 2314-2332. https://doi.org/10.1016/j.ecolmodel.2011.01.003

Ogilvie, J. (2017). Flow-Line and Wet-Areas Conformance Testing of Wetland Locations Using LiDAR and SRTM Elevation Data. Fredericton, New Brunswick: University of New Brunswick.

Prose, D. V. (1985). Persisting Effects of Armored Military Maneuvers Some Soils of the Mojave Desert. Environmental Geology and Water Sciences, 7, 163-170. https://doi.org/10.1007/BF02528800

Raven, P. H., Evert, R. F., \& Eichgorn, S. E. (1999). Biology of Plants (6th ed.). New York: W. H. Freeman.

Salmivaara, A., Miettinen, M., Finér, L., Launiainen, S., Korpunen, H., Tuominen, S., Heikkonen, J., Nevalainen, P., Sirén, M., Ala-Ilomäki, J., \& Uusitalo, J. (2018). Wheel Rut Measurements by Forest Machine-Mounted LiDAR Sensors-Accuracy and Potential for Operational Applications? International Journal of Forest Engineering, 29, 1-12. https://doi.org/10.1080/14942119.2018.1419677

Shmulevich, I., Mussel, U., \& Wolf, D. (1998). The Effect of Velocity on Rigid Wheel Performance. Journal of Terramechanics, 35, 189-207.

Startsev, A., \& McNabb, D. (2000). Effects of Skidding on Forest Soil Infiltration in West-Central Alberta. Canadian Journal of Soil Science, 80, 617-624. https://doi.org/10.4141/S99-092

Taghavifar, H., \& Mardani, A. (2014). Effect of Velocity, Wheel load and Multipass on Soil Compaction. Journal of the Saudi Society of Agricultural Sciences, 13, 57-66. 
https://doi.org/10.1016/j.jssas.2013.01.004

Vega-Nieva, D. J. D., Murphy, P. N. C., Castonguay, M., Ogilvie, J., \& Arp, P. A. (2009). A Modular Terrain Model for Daily Variations in Machine-Specific Forest Soil Trafficability. Canadian Journal of Soil Science, 89, 93-109. https://doi.org/10.4141/CJSS06033

White, B., Ogilvie, J., Campbell, D. M. H., Hiltz, D., Gauthier, B., Chisholm, H. K. H., Wen, H. K., Murphy, P. N. C., \& Arp, P. A. (2012). Using the Cartographic Depth-to-Water Index to Locate Small Streams and Associated Wet Areas across Landscapes. Canadian Water Resources Journal, 37, 333-347. https://doi.org/10.4296/cwrj2011-909 


\section{Appendix 1}

Normalized clearance histograms perharvest block by machine type, with best-fitted Gamma distribution functions overlaid.

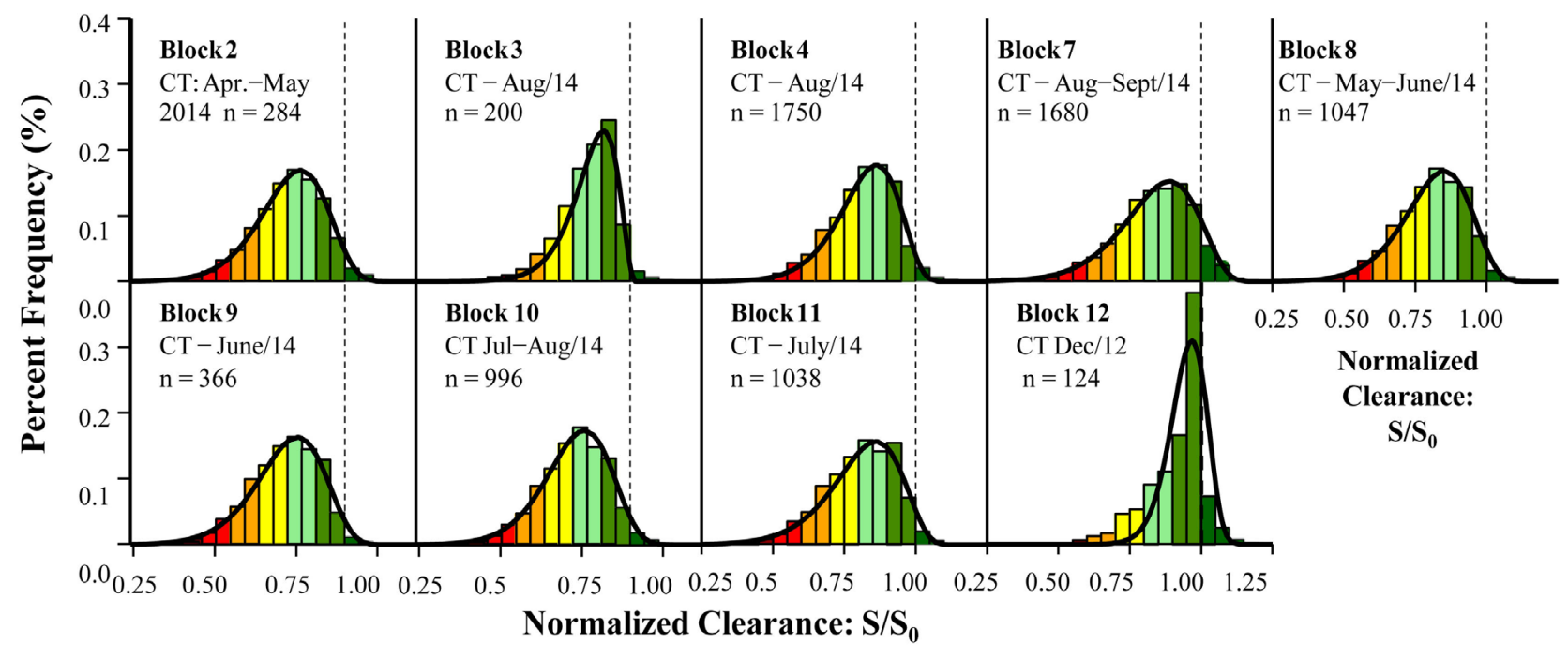

Figure A1-1. Histograms for the JD11 normalized clearance data, by block. Normalized clearance in terms of chassis- to sensor-to solid-ground distances: $\mathrm{M} 0 / \mathrm{S} 0=0.57$.

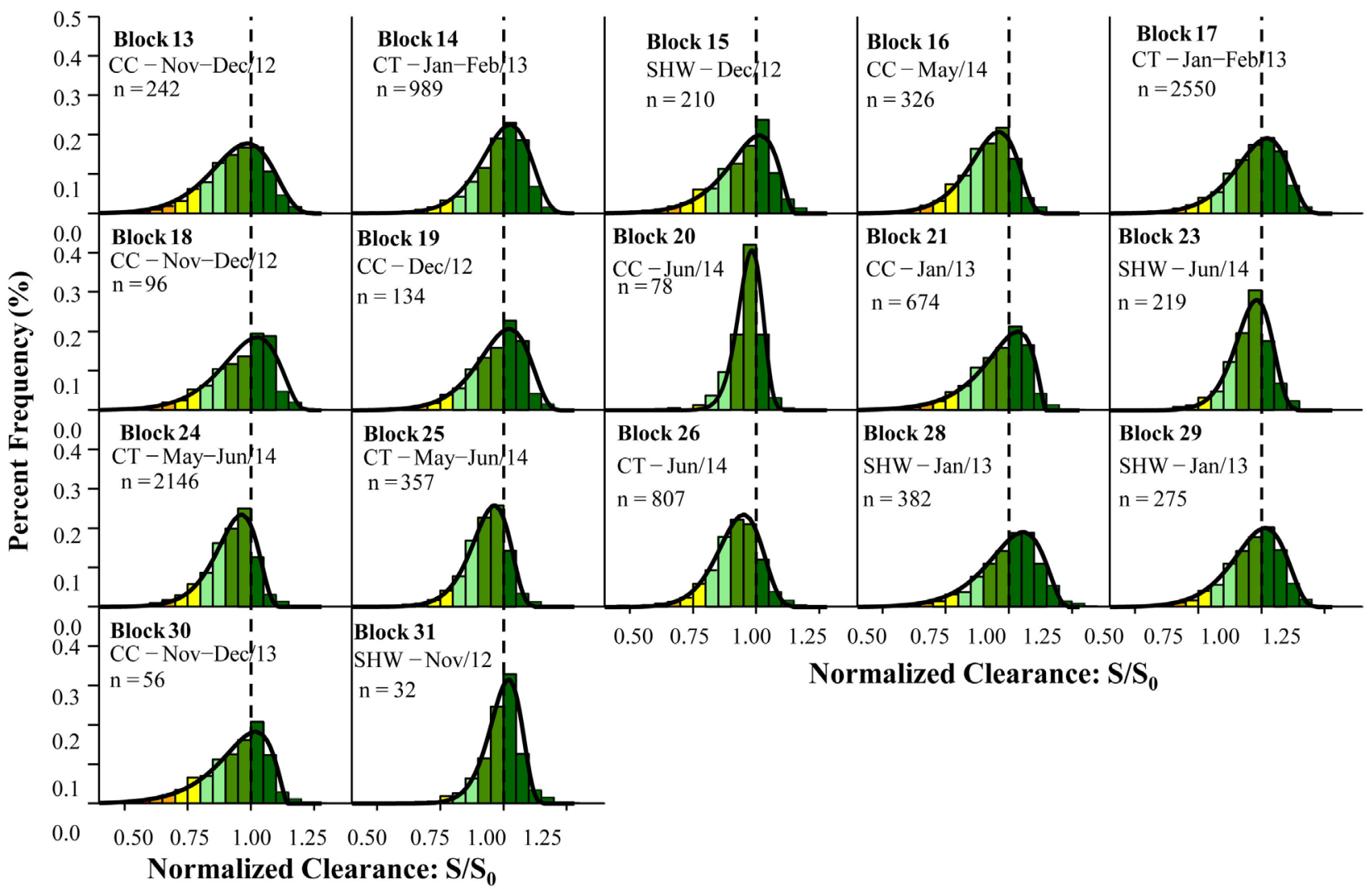

Figure A1-2. Histograms for the JD15 normalized clearance data, by block. Normalized clearance in terms of chassis- to sensor-to solid-ground distances: $\mathrm{M} 0 / \mathrm{S} 0=0.57$. 


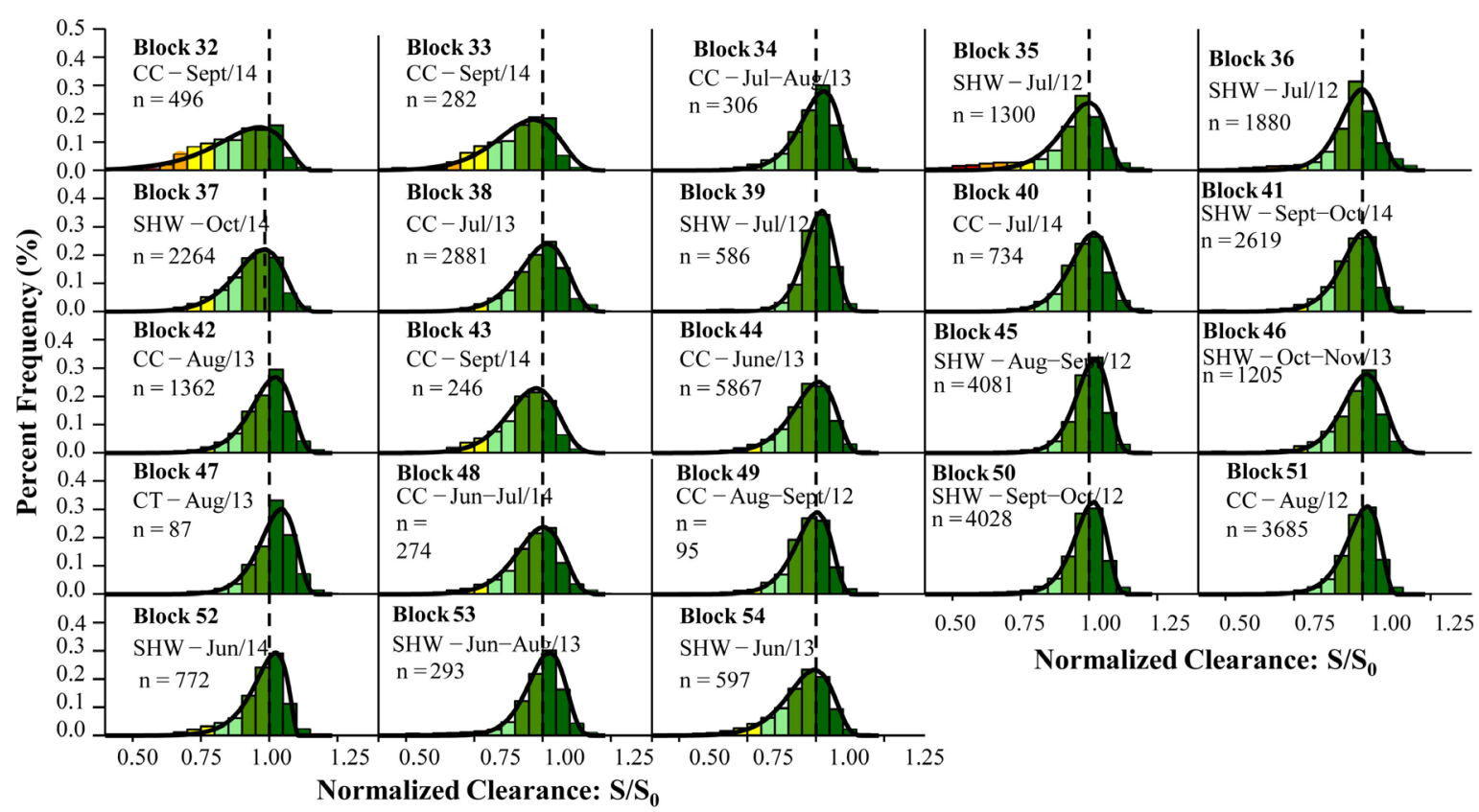

Figure A1-3. Histograms for the TC normalized clearance data, by block. Normalized clearance in terms of chassis- to sensor- to solid-ground distances (front): $\mathrm{M} 0 / \mathrm{S} 0=0.63$.

\section{Appendix 2}

$10^{\text {th }}, 25^{\text {th }}, 50^{\text {th }}, 75^{\text {th }}$ and $90^{\text {th }}$ percentiles for the $\leq 1$ normalized clearance data per block and machinetype by pass number, speed and DTW classes.

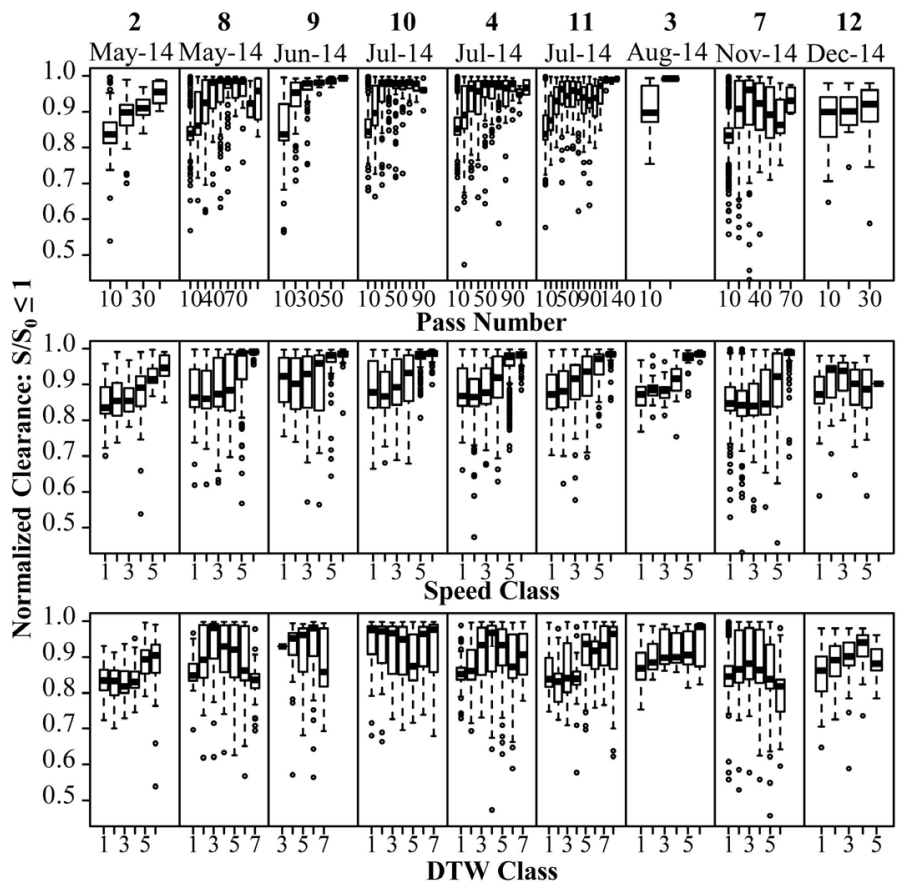

Figure A2-1. Normalized JD11 clearance $\leq 1$ box plots by pass number, speed class, and DTW classes per block. Blocks \#1, \#5 and \#6 generated no clearance data due to sensor malfunction. Normalized clearance in terms of chassis- to sensor-to solid-ground distances: $\mathrm{M} 0 / \mathrm{S} 0=0.57$. 


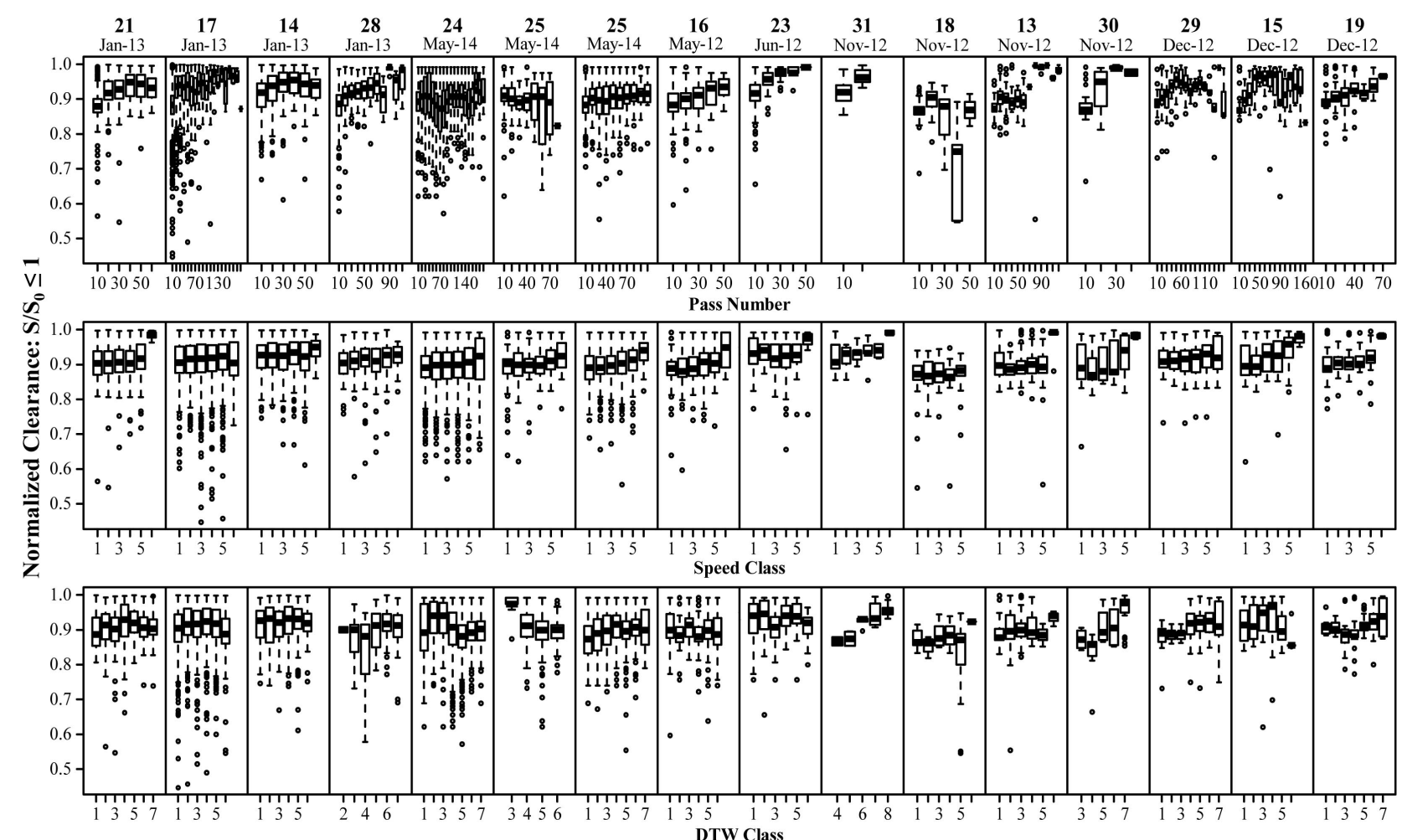

Figure A2-2. Normalized JD15 clearance $\leq 1$ box plots by pass number, speed class, and DTW classes per block. Blocks \#32 generated no clearance data due to sensor malfunction. Normalized clearance in terms of chassis- to sensor-to solid-ground distances: $\mathrm{M} 0 / \mathrm{S} 0=0.55$.

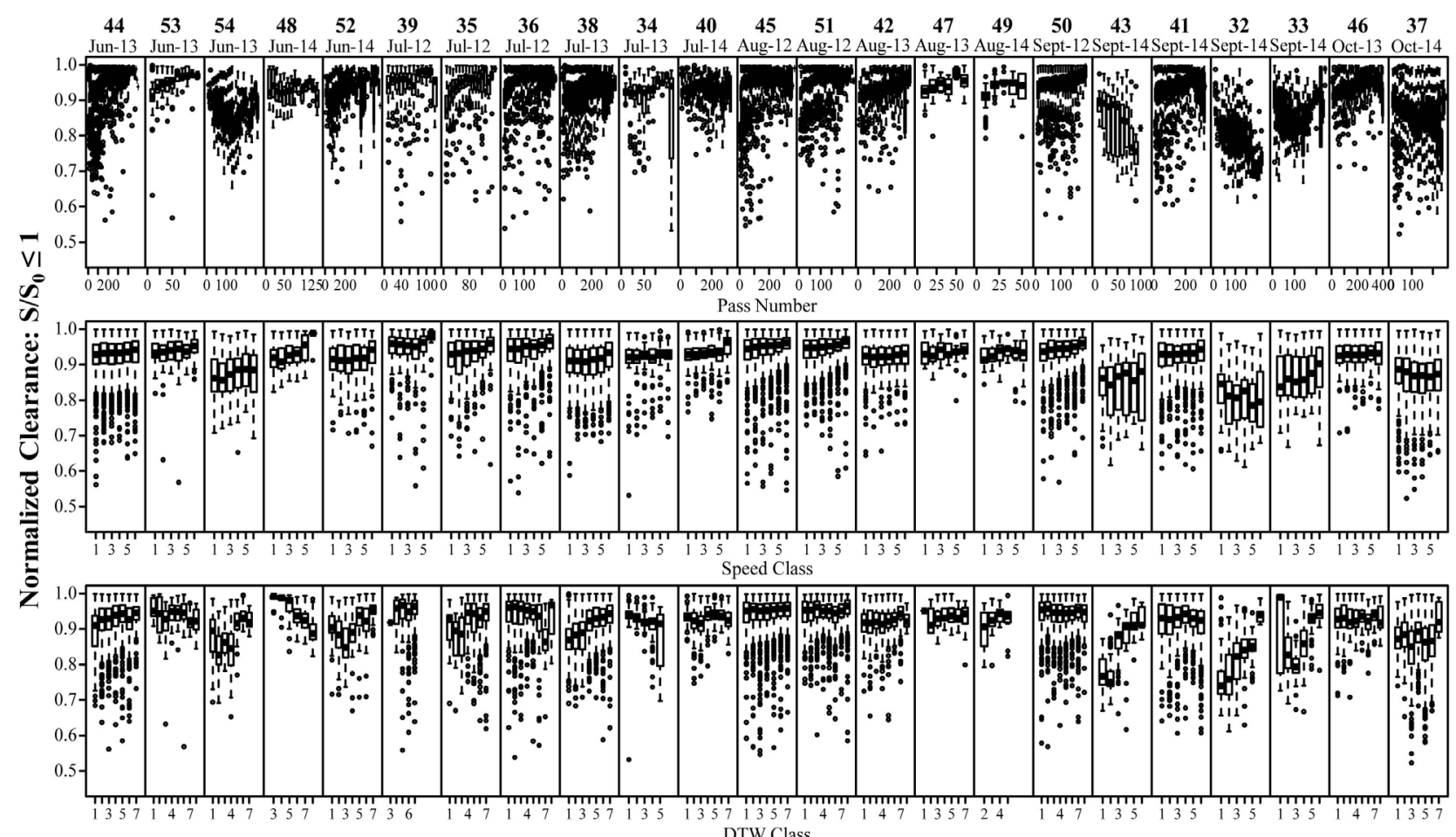

Figure A2-3. Normalized TC clearance $\leq 1$ percentile box plots by pass number, speed class, and DTW classes per block. Normalized clearance in terms of chassis- to sensor-to solid-ground distances: $\mathrm{M} 0 / \mathrm{S} 0=0.63$. 


\section{Appendix 3}

Year-round hydrological weather and ground conditions 2011-2014.
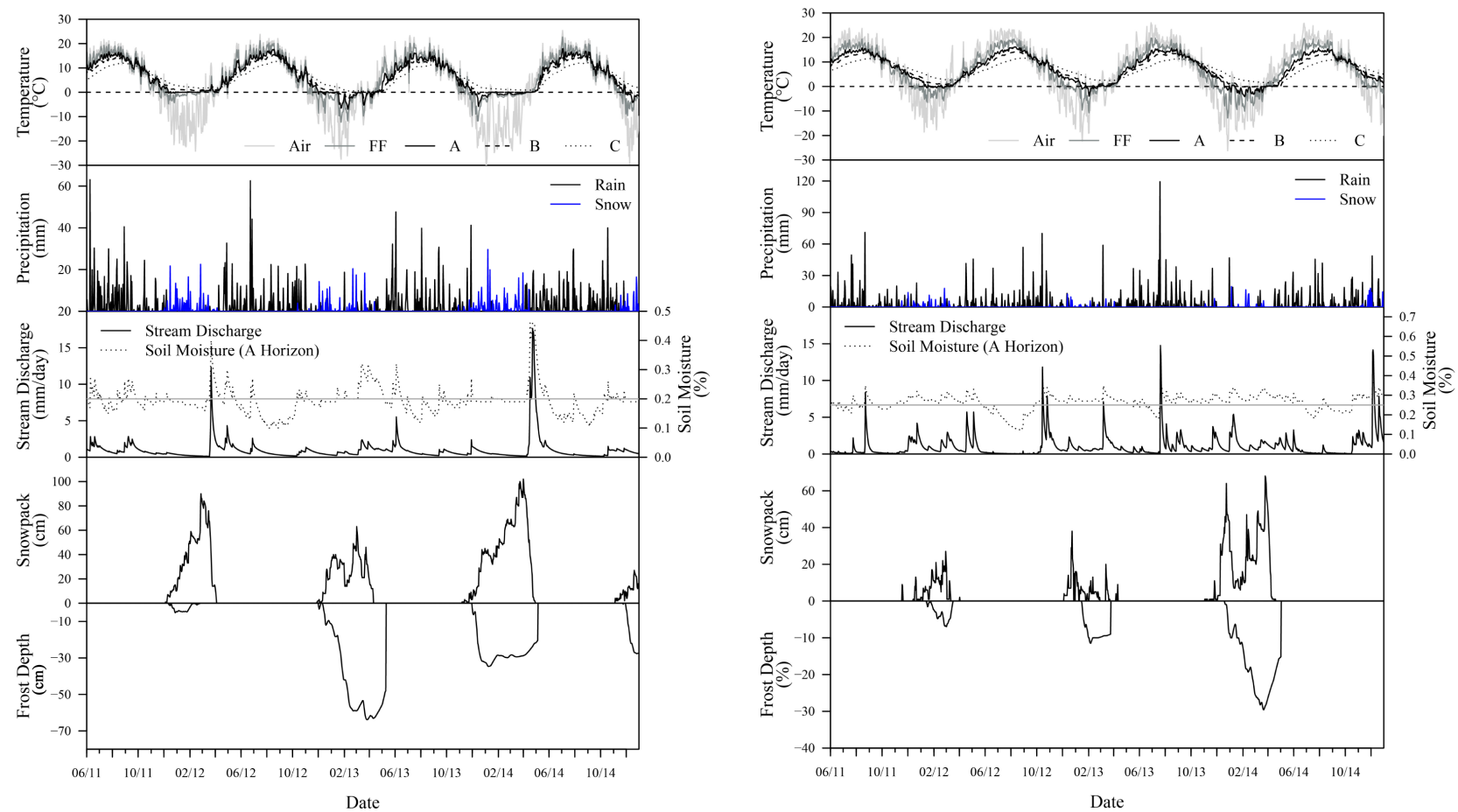

Figure A3-1. Daily variations in air temperature and precipitation, with modelled stream discharge, volumetric soil moisture content including field capacities for the wider NWU (left) and MWU + LL (right) areas, and snowpack depth, frost depth, and volumetric soil moisture content for the wider NWU (left) and MWU + LL (right) areas. 